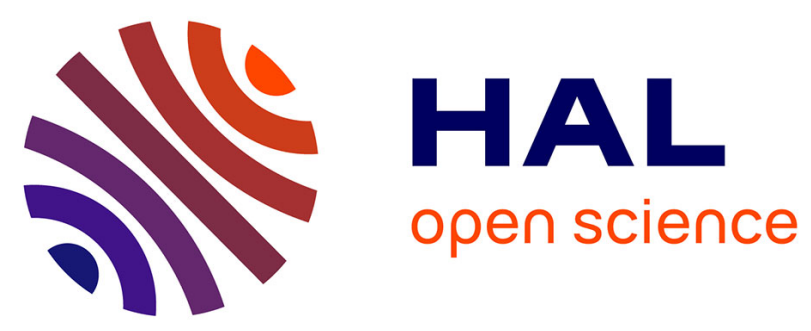

\title{
Conservation, traitement et consommation des produits végétaux dans la grotte chalcolithique de Foissac (Aveyron): les données carpologiques
}

Laurent Bouby, Philippe Marinval

\section{To cite this version:}

Laurent Bouby, Philippe Marinval. Conservation, traitement et consommation des produits végétaux dans la grotte chalcolithique de Foissac (Aveyron): les données carpologiques. Gallia Préhistoire - Archéologie de la France préhistorique, 2005, 47, pp.147-165. 10.3406/galip.2005.2048 . hal02346144

HAL Id: hal-02346144

https://hal.science/hal-02346144

Submitted on 21 Jan 2020

HAL is a multi-disciplinary open access archive for the deposit and dissemination of scientific research documents, whether they are published or not. The documents may come from teaching and research institutions in France or abroad, or from public or private research centers.
L'archive ouverte pluridisciplinaire HAL, est destinée au dépôt et à la diffusion de documents scientifiques de niveau recherche, publiés ou non, émanant des établissements d'enseignement et de recherche français ou étrangers, des laboratoires publics ou privés.

\section{(ㅇ)(1) $\$$}

Distributed under a Creative Commons Attribution - NonCommercial - NoDerivatives 44.0 


\title{
CONSERVATION, TRAITEMENT ET CONSOMMATION DES PRODUITS VÉGÉTAUX DANS LA GROTTE CHALCOLITHIQUE DE FOISSAC (AVEYRON)
}

\section{Les données carpologiques}

\author{
Laurent BoubY ${ }^{*}$ et Philippe MARVINVAL ${ }^{* *}$
}

\begin{abstract}
Mots-clés. Chalcolithique, carpologie, agriculture, stockage, traitement des récoltes, lin, rongeurs, imbibition.
Résumé. Deux secteurs de la grotte chalcolithique de Foissac ont livré des fruits et graines archéologiques. Dans le premier secteur, dit du Chaos, des carporestes carbonisés étaient visibles à l'œil nu lors des fouilles. En deux endroits, les semences ont été supposées provenir de deux vases brisés sur place qui, dans cette hypothèse, auraient pu contenir des réserves végétales. L'étude carpologique a montré que des produits secondaires du décorticage de lamidonnier (Triticum dicoccum), principalement composés de vannes, étaient réservés dans le premier vase. Par leur hauteur de croissance, les mauvaises herbes associées ont permis de supposer que la moisson était effectuée par une coupe haute. Le second vase de stockage contenait une réserve d'amidonnier, probablement vieille de plus d'une année car contaminée par une grande quantité de déjections de rongeurs. Notre analyse montre l'existence d'un troisième produit végétal : une réserve de graines de lin (Linum usitatissimum), probablement issue d'un petit vase. La mention du lin est intéressante car la culture de cette plante oléagineuse et textile n'avait jamais été prouvée jusqu'à présent dans le sud et l'ouest de la France avant l'âge du Bronze. Dans le secteur de la Rivière, les niveaux subaquatiques anaérobies ont permis la conservation de restes végétaux non carbonisés. Malheureusement, l'usage d'un protocole de tamisage et de tri mal adapté aux recherches carpologiques a conduit à la perte de nombreuses informations. L'étude de ce second secteur procure néanmoins de précieux renseignements sur une gamme de fruits sauvages cueillis.
\end{abstract}

Key-words. Chalcolithic, archaeobotany, agriculture, storage, crop processing, linseed, rodent, waterlogging.

Abstract. Two areas of the Foissac Chalcolithic cave delivered archaeobotanical fruit and seed remains. In the Chaos area, carbonized seeds were visible to the naked eye during excavations. In two places they had been supposed to be connected to two in situ broken vessels, which therefore could have contained crop storages. The archaeobotanical study shows that emmer (Triticum dicoccum) dehusking byproducts, principally composed of chaff, were located in the first vessel. Associated weeds are leading to the hypothesis of a high level of reaping of emmer. The second storage ceramic was enclosing an ancient storage of emmer, largely contaminated by rodent droppings. Our analyse gives evidence for a third product : a linseed (Linum usitatissimum) reserve, probably stored in a small vessel. This record is of some interest because Neolithic or Chalcolithic linseed growing had never been proven up to now in Southern and Western France. The anaerobic, underwater levels of the Rivière area allowed preservation of uncarbonized plant remains. Unfortunately, sieving and sorting methods were not accurately adapted to archaeobotanical sampling. A lot of information is therefore missing. However this second area is bringing noticeable data on a range of wild collected fruits.

* Centre d'études Préhistoire, Antiquité, Moyen Âge, CNRS, UMR 6130, Sophia Antipolis, 250 rue Albert-Einstein, F-06560 Valbonne. Courriel : bouby@cepam.cnrs.fr.

** Centre d'anthropologie, CNRS, UMR 8555, Université Paul-Sabatier, 39 allées Jules-Guesde, F-31000 Toulouse. Courriel : marinval@cict.fr. 
Schlagwörter. Chalkolithikum, Botanische Makrorestanalyse, Ackerbau, Vorratshaltung, Behandlung der Ernte, Lein, Nagetiere, Feuchterhaltung.

Zusammenfassung. Zwei Abschnitte der chakolithischen Höhle von Foissac haben archäologische Überreste von Früchten und Körnern erbracht. Im ersten Höhlenabschnitt, dem sogenannten Versturz, waren verkohlte Pflanzenreste bereits während der Ausgrabung mit bloßem Auge sichtbar. Von zwei Stellen bestand der Verdacht, daß die Sämereien aus zwei dort zerbrochenen Gefäßen stammten die dieser Hypothese zufolge vegetabile Vorräte enthalten haben mußten. Wie die Makrorestanalyse zeigt, waren in dem ersten Gefäß beim Schälen von Emmer (Triticum dicoccum) angefallene Sekundärprodukte (vor allem Hülsen) deponiert. Die Wuchshöhe der mitgefundenen Unkräuter erlaubt die Vermutung daß die Ernte durch hohen Schnitt ausgeführt wurde. Das zweite Vorratsgefäß enthielt einen Emmervorrat der aufgrund des hohen Anteils von Nagerkot wahrscheinlich bereits älter als ein Jahr war. Unsere Untersuchung weist darüberhinaus die Existenz eines dritten vegetabilen Produktes nach, nämlich eines wahrscheinlich aus einem kleinen Gefäß stammenden Vorrats an Leinsamen (Linum usitatissimum). Das Auftreten von Lein ist interessant weil die Kultur dieser Öl- und Faserpflanze bislang für Südostfrankreich vor der Bronzezeit nicht belegt war. In dem Höhlenabschnitt, der vom Fluß durchzogen wird, konnten in den unter Wasser gelegenen Fundschichten unverkohlte Pflanzenreste unter Luftabschluß erhalten bleiben. Da die Probenwahl und der Ablauf des Siebvorgangs aber schlecht auf die Makrorestanalyse abgestimmt waren, kam es leider zum Verlust vieler Informationen. Die Untersuchung dieses zweiten Abschnittes erbrachte dennoch wertvolle Erkenntnisse zu einer Auswahl gesammelter Wildfrüchte.

Übersetzung: Stefan WIRTH

La grotte étudiée se situe sur la commune de Foissac (Aveyron), au nord-est du causse de Limogne, à une quinzaine de kilomètres au nord de Villefranche-deRouergue, en direction de Figeac (fig. 1). Le réseau karstique débute avec la perte de la Jonquière, petit affluent $\mathrm{du}$ Lot, qui poursuit son cours souterrain sur près de $10 \mathrm{~km}$.

Des fouilles archéologiques ont été conduites entre 1977 et 1988, par S. Cours, H. Duday, A. du Fayet de la Tour, M.-A. Garcia et F. Rouzaud, dans le but d'étudier les diverses activités exercées par les hommes du Chalcolithique à l'intérieur de la cavité (Cours et al., 1984; Cours et al., inédit ; Garcia et al., 1987; Garcia, Rouzaud, 1994). Différents éléments témoignent de l'ancienneté de la fréquentation du réseau par les hommes, au cours du Paléolithique moyen et final. En revanche, il n'existe aucun témoin d'une présence humaine postérieure au Chalcolithique; la cavité semble dès cette époque scellée par l'effondrement du porche et restera d'accès extrêmement difficile jusqu'au percement d'une entrée artificielle et l'aménagement pour les visites à partir de 1965 .

L'occupation chalcolithique s'est concentrée dans les quelque $300 \mathrm{~m}$ de galerie qui succèdent à l'entrée souterraine de la rivière. Cette partie est composée de salles parmi les plus grandes du réseau. Les recherches ont tout d'abord concerné les aménagements, carrières et traces liés à l'extraction d'argile, répartis en divers points de la grotte, ainsi que les restes humains, en particulier 4 sépultures individuelles. Conjointement à ces travaux, deux zones ont donné lieu à des fouilles spatiales : les secteurs de la Rivière et du Chaos (fig. 2). Tous deux ont révélé la présence de restes végétaux.
Grâce au pompage et au détournement de la Jonquière en période de basses eaux, des fouilles ont pu être conduites sur l'ancien lit de la rivière. Les deux niveaux chalcolithiques subaquatiques se sont révélés très favorables à la conservation des restes végétaux, avec notamment la présence de très nombreux fragments de bois non carbonisés, dont certains exemplaires façonnés. Seize bases de poteaux en bois ont été découvertes, formant deux alignements orientés selon l'axe du ruisseau. Les poteaux sont supposés avoir soutenu une structure en bois, tablier de pont ou plus probablement plancher, attesté sur près de $70 \mathrm{~m}^{2}$ mais dont la surface était sans doute bien supérieure (Cours et al., 1984 et inédit ; Garcia et al., 1987). Il semble que cet édifice ait connu des réparations, voire une réfection totale.

Le secteur du Chaos se situe à quelque 25 mètres de l'entrée préhistorique de la grotte. Il s'agit d'un éboulis de blocs rocheux dominant la rivière souterraine. En hiver ou à la suite des orages estivaux, la Jonquière entre en crue, inondant la grotte et entraînant le soutirage des couches archéologiques entre les blocs du Chaos. De ce fait, les fouilles ont dû se limiter à quelques lambeaux de couches en place ou à des ensembles soutirés en bloc entre les rochers. Au bas de la stratigraphie, une première couche d'argile jaune, compacte et pratiquement dénuée de mobilier archéologique, recouvre les blocs. L'argile fut rapportée par les occupants - peut-être en provenance des carrières de la grotte - et constitue un aménagement du Chaos rocheux. Au-dessus s'observe une seconde couche archéologique, également chalcolithique, très cendreuse et 


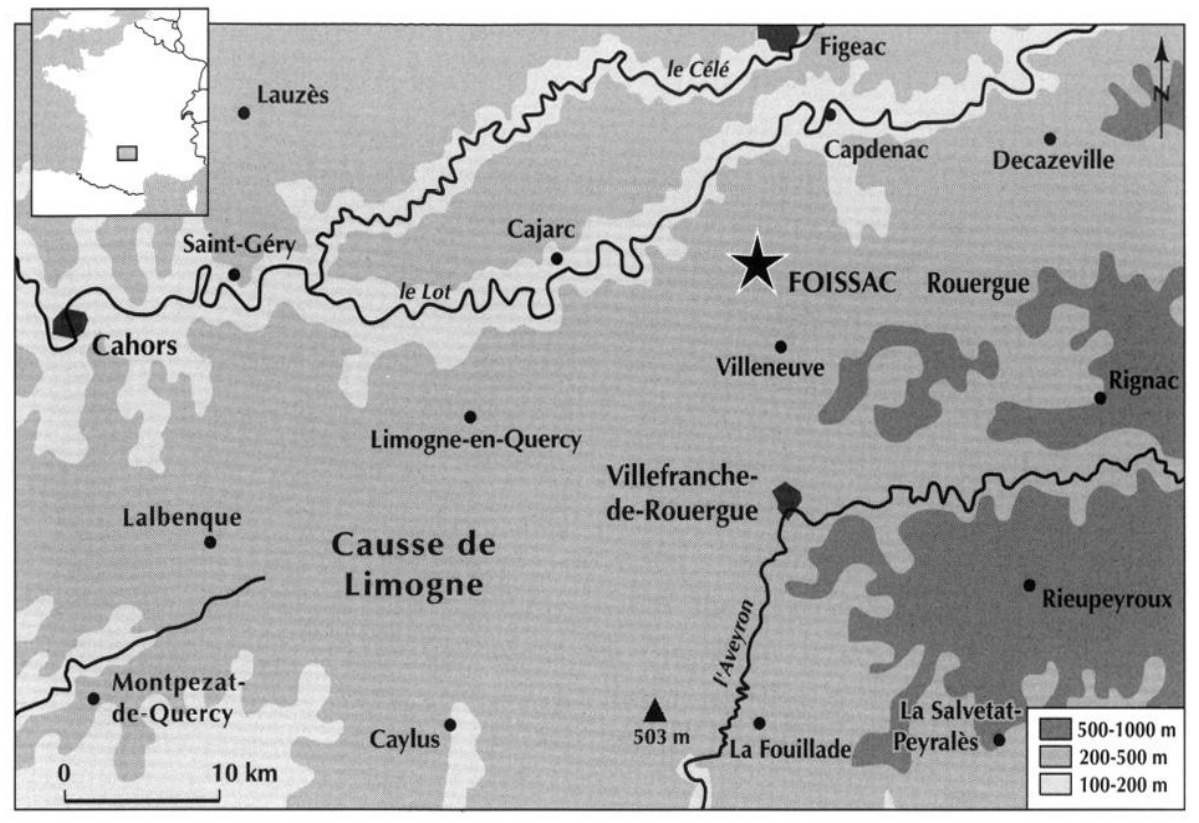

Fig. 1 - Localisation de la grotte de Foissac, Aveyron (fond de carte (iéoAtlas).

riche en charbons de bois, semences carbonisées, fragments d'argile cuite, tessons de céramique, mobilier lithique, éléments de parure (perles en particulier) et ossements animaux.

Les nodules d'argile durcie au feu, très nombreux, sont constitués uniquement d'argile naturelle et portent fréquemment, sur une ou plusieurs faces, des empreintes diverses: traces de doigts, éléments de vannerie, épis, semences, tiges et feuilles de plantes herbacées de type graminées. Leur cuisson, franchement irrégulière, semble d'origine accidentelle. En de multiples endroits les blocs rocheux sont rubéfiés et le mobilier archéologique porte également parfois des traces de chauffe. Les fouilleurs émettent l'hypothèse d'un incendie ayant ravagé une structure, probablement en bois, aménagée sur le Chaos. Celle-ci pourrait être en continuité avec l'aménagement voisin du secteur de la Rivière.

L'aménagement du Chaos a pu avoir une fonction de stockage ou de réserve. Cette hypothèse est notamment appuyée par l'existence de plusieurs vases brisés in situ. Deux grands vases de stockage en particulier semblaient en relation avec des concentrations de semences carbonisées. L'étude archéozoologique conclut par ailleurs à la conservation de produits carnés à l'intérieur de la grotte

Fig. 2 - Principaux secteurs de recherche dans la grotte de Foissac (d'après Garcia et al., 1987).

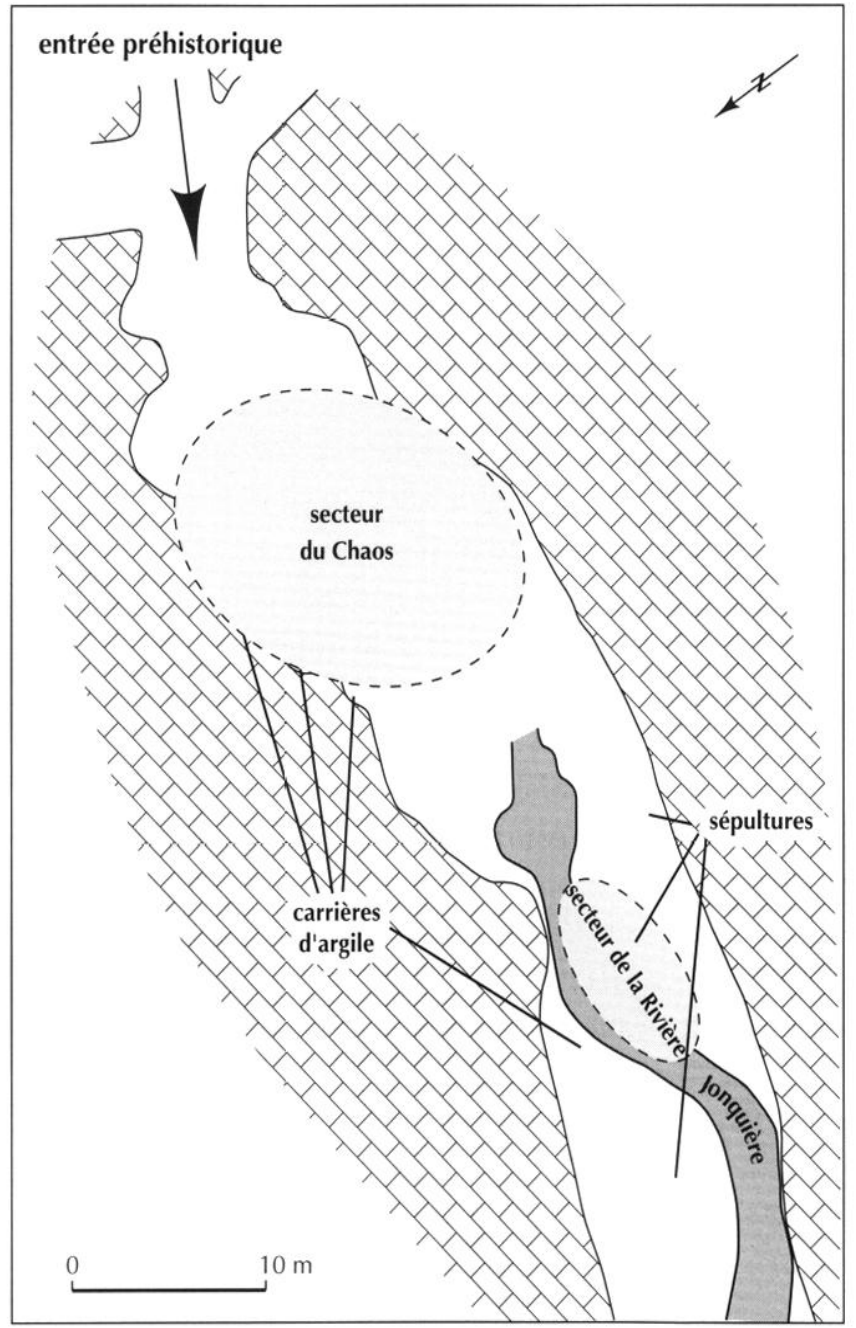


(Lignereux et al., 1994). L'abondance des éléments de parures s'accorderait également avec l'existence d'une réserve ou d'une cachette dans la cavité (Garcia et al., 1987 ; Cours et al., inédit).

L'occupation chalcolithique de la grotte cessa brutalement, sans doute à la suite de l'incendie, puis, peu de temps après, le porche d'entrée s'est effondré, scellant la cavité pour une très longue période. Le mobilier archéologique, bien plus abondant dans le secteur du Chaos que dans celui de la Rivière, est très cohérent et caractéristique du Chalcolithique. Il se compose notamment de vases en céramique, entiers ou dont la forme peut être reconstituée, de nombreuses armatures de flèches, de grattoirs, lames et autres pièces lithiques, d'une douzaine de meules en granite, de quelques outils en os (poinçons, ciseau) et d'éléments de parure variés (pendeloques, boutons, perles, dentales). Les neuf datations radiocarbone publiées (Cours et al., 1984; Jaubert, 1996) possèdent malheureusement pour plusieurs d'entre elles un écart-type important; elles permettent néanmoins de confirmer que l'occupation de la grotte se déroule dans le courant du troisième millénaire, en concordance avec le mobilier.

Les secteurs de la Rivière et du Chaos offrent des perspectives carpologiques riches et complémentaires. De par ses conditions de conservation anaérobies, le secteur de la Rivière apparaît favorable à l'enregistrement d'un nombre élevé de taxons, potentiellement fertile en informations détaillées sur l'environnement et les plantes exploitées. Possible lieu de stockage, le secteur du Chaos semble plutôt à même de documenter les activités de production et de traitement des produits végétaux. Ces perspectives de recherche paraissent toutefois quelque peu obscurcies par la variabilité des techniques d'échantillonnage et de traitement des prélèvements, ainsi que par la conservation erratique de la couche charbonneuse dans le secteur du Chaos.

\section{MATÉRIEL ET MÉTHODES}

Au cours des fouilles, les sédiments de la Rivière et du Chaos furent intégralement tamisés, dans le but principal de rechercher le petit mobilier archéologique ainsi que les restes malacologiques et de micromammifères. Une station de tamisage équipée d'une série de tamis aux mailles échelonnées de $25 \mathrm{~mm}$ à $0,6 \mathrm{~mm}$ a été installée directement dans la grotte, fonctionnant avec l'eau du ruisseau. Après tamisage sous eau, les divers restes étaient immédiatement triés à l'ceil nu par l'équipe archéologique. Il est évident que ce procédé implique une certaine sélection des carporestes les plus gros et les plus facilement identifiables pour un observateur non spécialisé. L'intégralité des semences étudiées de la Rivière a été recueillie selon ce protocole, ainsi qu'une partie des échantillons du Chaos (30\%).

Dans ce secteur, l'abondance des semences carbonisées a rapidement conduit les fouilleurs à effectuer des prélèvements spécifiques, afin qu'ils soient traités selon les méthodes les mieux adaptées. Nous avons donc eu la possibilité de tamiser 10 prélèvements par flottation manuelle, sur cribles de $2 \mathrm{~mm}$ et $0,5 \mathrm{~mm}$, puis de les trier sous loupe binoculaire au laboratoire de l'unité de carpologie du Centre d'anthropologie (UMR 8555, Toulouse). Dans un second temps, plusieurs années après la clôture du chantier, quatre prélèvements supplémentaires ont été effectués dans le but d'évaluer la densité carpologique du sédiment (nombre de restes/litre). L'ensemble des prélèvements étudiés provient de la couche cendreuse et charbonneuse située au-dessus de la couche d'argile. Le secteur était découpé selon un carroyage de $1 \mathrm{~m}$ de côté et tous les prélèvements sont issus de $20 \mathrm{~m}^{2}$ différents et juxtaposés.

En outre, un ensemble d'empreintes végétales dans des fragments d'argile cuite du Chaos a été étudié grâce à la réalisation de moulages, à l'aide d'un élastomère silicone à rétraction nulle (RTV 573 A).

\section{RÉSULTATS}

\section{LE SECTEUR DU CHAOS}

Les vingt échantillons, ou prélèvements, étudiés dans le secteur du Chaos ont livré un total de 3722 carporestes carbonisés, conduisant à l'identification de 22 taxons au minimum (tabl. I). Un nombre important de semences non carbonisées de sureau (Sambucus elnulus en particulier) a été recueilli dans plusieurs échantillons. Bien que leur conservation puisse être liée à l'imbibition par les eaux de la Rivière, dans le doute ces restes sont considérés comme des pollutions. Aux informations relatives au matériel carbonisé s'ajoute l'analyse de 19 empreintes dans l'argile cuite ayant permis l'identification de deux céréales, l'orge polystique nue (Hordeum vulgare var. nudum) et l'amidonnier (Triticum dicoccum) (tabl. II).

La fragmentation des semences, qui oscille entre 0 et $81 \%$, est plus particulièrement élevée dans les bandes I, J et K 
Tabl. I - Résultats carpologiques du secteur du Chaos de la grotte de Foissac, restes carbonisés.

\begin{tabular}{|c|c|c|c|c|c|c|c|c|c|c|c|c|c|c|c|c|c|c|c|c|c|c|c|}
\hline $\begin{array}{l}\text { Carré } \\
\text { Volume tamisé en ml } \\
\text { Prélèvement spécifique carpologie }\end{array}$ & & G19 & $\begin{array}{c}120 \\
150 \\
X\end{array}$ & $\begin{array}{c}220 \\
- \\
\mathrm{x}\end{array}$ & $\begin{array}{c}120 \\
500 \\
X\end{array}$ & $\begin{array}{c}121 \\
- \\
x\end{array}$ & $\begin{array}{c}\text { K19 } \\
\text { X }\end{array}$ & $\begin{array}{c}\text { K20 } \\
- \\
\mathrm{x}\end{array}$ & $\begin{array}{c}\mathrm{K} 21 \\
- \\
\mathrm{x}\end{array}$ & $\begin{array}{c}\text { L19 } \\
- \\
\mathrm{x}\end{array}$ & $\begin{array}{c}120 \\
- \\
x\end{array}$ & $\begin{array}{c}\text { L21 } \\
: \\
\mathrm{x}\end{array}$ & $\begin{array}{c}\text { M19 } \\
\\
\mathrm{x}\end{array}$ & $\begin{array}{c}\text { M19 } \\
300 \\
X\end{array}$ & $\begin{array}{c}\text { M20 } \\
- \\
\mathrm{X}\end{array}$ & M22 & $\begin{array}{c}\text { N19 } \\
-\end{array}$ & N20 & $\begin{array}{c}\text { N21 } \\
-\end{array}$ & X95 & $\begin{array}{c}x \\
400 \\
X\end{array}$ & Total & $\begin{array}{c}\text { Ubiquité } \\
\%\end{array}$ \\
\hline 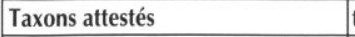 & type reste & & & & & & & & & & & & & & & & & & & & & & \\
\hline \multicolumn{24}{|l|}{ Plantes cultivées - semences } \\
\hline \multirow[t]{2}{*}{ Cerealia } & fg. & - & 14 & 50 & 2 & 15 & 2 & - & 19 & 1 & - & - & 9 & 32 & 2 & - & - & - & - & - & 3 & 149 & 50 \\
\hline & fg. pré-carbo & - & - & - & - & - & - & - & - & 2 & - & - & 23 & 15 & - & - & - & - & - & - & - & 40 & 11 \\
\hline \multirow[t]{2}{*}{ Hordeum vulgare var. nudum } & \begin{tabular}{|l|l} 
sem. & \\
\end{tabular} & - & 2 & 18 & - & 4 & 1 & 1 & 9 & 1 & 3 & 1 & 3 & 1 & 2 & 3 & 4 & 1 & - & - & - & 54 & 78 \\
\hline & fg. & - & 1 & 3 & - & 2 & - & - & 5 & 2 & 1 & - & 7 & 1 & 2 & - & - & - & - & - & - & 24 & 44 \\
\hline Triticum aestivum/turgidum & sem. & - & - & 8 & - & 7 & - & - & 6 & - & 2 & 3 & 4 & 1 & 5 & 1 & 1 & - & 1 & - & - & 39 & 55 \\
\hline \multirow[t]{2}{*}{ Triticum dicoccum } & sem. & 1 & 2 & 65 & - & 80 & 8 & 9 & 152 & 6 & 62 & 4 & 206 & 158 & 34 & 8 & 4 & 12 & 2 & 1 & 5 & 819 & 100 \\
\hline & fg. & - & 2 & 49 & 1 & 33 & 3 & - & 96 & 15 & 16 & 3 & 35 & 10 & 11 & - & - & - & - & - & 1 & 275 & 61 \\
\hline \multirow[t]{2}{*}{ Triticum monococcum } & sem. & - & - & - & - & - & - & - & 2 & - & 1 & 1 & 3 & - & - & - & - & - & - & - & - & 7 & 22 \\
\hline & fg. & - & - & - & - & 1 & - & - & - & - & - & - & - & - & - & - & - & - & - & - & - & 1 & 5 \\
\hline Triticum sp. & fg. & - & 1 & 198 & 1 & 230 & 45 & - & 192 & 34 & 41 & 15 & $\mid 157$ & 71 & 30 & 2 & 3 & - & 1 & 1 & 11 & 1033 & 83 \\
\hline \multirow{2}{*}{ Linum usitatissimum } & sem. & - & - & 2 & - & 86 & - & 2 & 2 & 2 & - & 1 & -1 & - & - & - & - & - & - & - & - & 95 & 33 \\
\hline & fg. & - & - & 1 & - & 42 & - & 8 & 2 & 1 & - & - & - & - & - & - & - & - & - & - & - & 54 & 28 \\
\hline Pisum sativum & sem. & - & - & - & - & - & - & - & - & - & - & 1 & - & - & - & - & - & - & - & - & - & 1 & 5 \\
\hline \multicolumn{24}{|l|}{ Plantes cultivées - vannes } \\
\hline Hordeum vulgare & entrenœud & - & - & - & - & 2 & - & - & - & - & - & - & 2 & - & - & - & - & - & - & - & - & 4 & 11 \\
\hline Linum usitatissimum & fg. capsule & - & - & 1 & - & - & - & - & - & - & - & - & - & - & - & - & - & - & - & - & - & 1 & 5 \\
\hline Triticum cf. aestivum s.l. & entrenœud & - & - & - & - & 1 & - & 2 & - & - & - & - & - & - & - & - & - & - & - & - & - & 3 & 11 \\
\hline Triticum dicoccum & furca & - & 1 & - & - & 2 & 1 & 2 & 4 & 15 & 3 & 1 & 135 & 63 & 17 & - & - & - & - & - & - & 244 & 55 \\
\hline & base glume & - & - & - & - & 6 & - & 4 & 2 & 6 & 1 & - & 13 & 39 & 18 & - & - & - & - & - & - & 89 & 39 \\
\hline Triticum monococcum & furca & - & - & - & - & 2 & - & - & - & 5 & 1 & - & 13 & 8 & 3 & - & - & - & - & - & - & 32 & 28 \\
\hline & base glume & - & - & - & - & - & - & - & - & 5 & - & - & 1 & - & 2 & - & - & - & - & - & - & 8 & 17 \\
\hline T. dicoccum/monococcum & furca & - & - & - & - & - & - & - & - & 3 & 4 & 1 & 4 & 18 & - & - & - & - & - & - & - & 30 & 22 \\
\hline & base glume & - & 2 & 5 & 2 & 10 & - & 6 & - & 17 & 6 & - & 88 & 218 & 55 & - & - & - & - & - & 5 & 414 & 50 \\
\hline & entrenœud & - & - & - & - & 2 & - & - & - & - & - & - & 3 & 34 & 2 & - & - & - & - & - & - & 41 & 17 \\
\hline Arbustes, buissons & & & & & & & & & & & & & & & & & & & & & & & \\
\hline Corylus avellana & fg. & - & - & 2 & - & 1 & 1 & - & 1 & - & - & 4 & 1 & - & 1 & - & - & - & - & - & - & 11 & 39 \\
\hline Sambucus ebulus & sem. & - & - & - & - & - & - & - & 2 & - & - & - & 4 & - & - & - & - & - & - & - & - & 6 & 11 \\
\hline Herbacées sauvages & & & & & & & & & & & & & & & & & & & & & & & \\
\hline Agrimonia eupatoria & capitule & - & - & - & - & - & - & - & - & - & - & - & 1 & - & - & - & - & - & - & - & - & 1 & 5 \\
\hline Ajuga sp. & sem. & & & & & & & & & & & & & & 1 & & & & & & & 1 & 45 \\
\hline Arrhenatherum elatius ssp. bulbosus t & tubercule & - & - & - & - & - & 1 & - & - & - & - & - & - & - & - & - & - & - & - & - & - & 1 & 5 \\
\hline & fg. & - & - & 1 & - & - & - & - & - & - & - & - & - & - & - & - & - & - & - & - & - & 1 & 5 \\
\hline Brassica sp. & sem. & - & - & - & - & - & - & - & - & - & - & - & - & - & 1 & - & - & - & - & - & - & 1 & 5 \\
\hline Brassicaceae & sem. & - & - & - & - & 1 & - & - & - & - & - & - & - & - & - & - & - & - & - & - & - & 1 & 5 \\
\hline Bromus hordeaceus/secalinus & sem. & - & - & - & - & 1 & - & - & - & - & - & - & 2 & - & - & - & - & - & - & - & - & 3 & 11 \\
\hline Camelina sativa & sem. & - & - & - & - & 10 & - & 1 & - & - & - & - & - & - & - & - & - & - & - & - & - & 11 & 11 \\
\hline Carex sp. & sem. & - & - & - & - & - & - & - & - & - & - & - & - & - & 1 & - & - & - & - & - & - & 1 & 5 \\
\hline Chenopodium album & sem. & - & - & - & - & 1 & - & 1 & - & - & - & 1 & - & - & - & - & - & - & - & - & - & 3 & 17 \\
\hline Cyperaceae & fg. tige & - & - & - & - & - & - & - & - & 2 & - & - & - & - & - & - & - & - & - & - & - & 2 & 5 \\
\hline Galium aparine/tricorne & sem. & - & - & - & - & - & - & - & - & - & - & - & 1 & - & - & - & - & - & - & - & - & 1 & 5 \\
\hline Galium cf. mollugo & sem. & - & - & - & - & - & - & - & - & - & - & - & - & - & 1 & - & - & - & - & - & - & 1 & 5 \\
\hline Galium sp. & sem. & - & - & - & - & - & - & - & - & 1 & - & - & 1 & - & - & - & - & - & - & - & - & 2 & 11 \\
\hline Phleum pratense & sem. & - & - & - & - & - & - & - & - & - & - & - & - & - & 3 & - & - & - & - & - & - & 3 & 5 \\
\hline Poaceae & sem. & - & - & - & - & 2 & - & - & - & - & 1 & 1 & 2 & - & - & - & - & - & - & - & - & 6 & 22 \\
\hline & fg. & - & - & 2 & - & - & - & 1 & - & 2 & - & - & - & 1 & - & - & - & - & - & - & - & 6 & 22 \\
\hline & fg. tige & - & - & - & - & - & - & - & 1 & - & - & - & 1 & 1 & - & - & - & - & - & - & - & 3 & 17 \\
\hline Polygonum aviculare & sem. & - & - & 1 & - & 1 & - & - & - & - & - & - & - & - & - & - & - & - & - & - & - & 2 & 11 \\
\hline Rumex cf. sanguineus & sem. & - & - & - & - & 1 & - & - & - & - & - & - & - & - & - & - & - & - & - & - & - & 1 & 5 \\
\hline Rumex sp. & fg. & - & - & - & - & - & - & - & 1 & - & - & - & - & - & - & - & - & - & - & - & - & 1 & 5 \\
\hline Vicia sp. & cotylédon & - & - & - & - & - & - & - & - & 1 & - & - & 1 & - & - & - & - & - & - & - & - & 2 & 11 \\
\hline Vicia/Lathyrus type & cotylédon & - & - & - & - & 2 & - & - & - & - & - & - & - & - & - & - & - & - & - & - & - & 2 & 5 \\
\hline Autres & & & & & & & & & & & & & & & & & & & & & & & \\
\hline Bourgeon & ind. & - & - & - & - & - & 1 & 1 & 2 & - & - & - & 1 & - & - & - & - & - & - & - & - & 5 & 22 \\
\hline Coprolithe rongeur & ind. & - & - & 32 & - & 121 & - & 2 & 22 & 1 & - & 1 & 2 & - & - & - & - & - & - & - & - & 181 & 39 \\
\hline Matière organique amorphe & fg. & - & - & $\cdot$ & - & 1 & - & - & 2 & - & - & - & 3 & - & - & - & - & - & $\cdot$ & - & - & 6 & 17 \\
\hline Total & & 1 & 25 & 438 & 6 & 667 & 63 & 40 & 522 & 122 & 142 & 38 & 726 & 671 & 191 & 14 & 12 & 13 & 4 & 2 & 25 & 3722 & \\
\hline Nombre de restes/litre & & $\cdot-1$ & 166,7 & - & 12 & - & - & - & - & - & - & - & 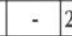 & 2236,7 & 7. & - & - & - & - & & 62,5 & $5 \cdot$ & \\
\hline Fragmentation $(\mathrm{Nb}$ fg/NR total $\times 100)$ & & 0,0 & 72,0 & 70,1 & 66,7 & 49,0 & 81,0 & 22,5 & 61,1 & 49,2 & 40,8 & 57,9 & 32,6 & \begin{tabular}{|l|}
19,5 \\
\end{tabular} & 24,1 & 14,3 & 25,0 & \begin{tabular}{|l|}
0,0 \\
\end{tabular} & 25,0 & 50,0 & 60,0 & 43,3 & \\
\hline
\end{tabular}




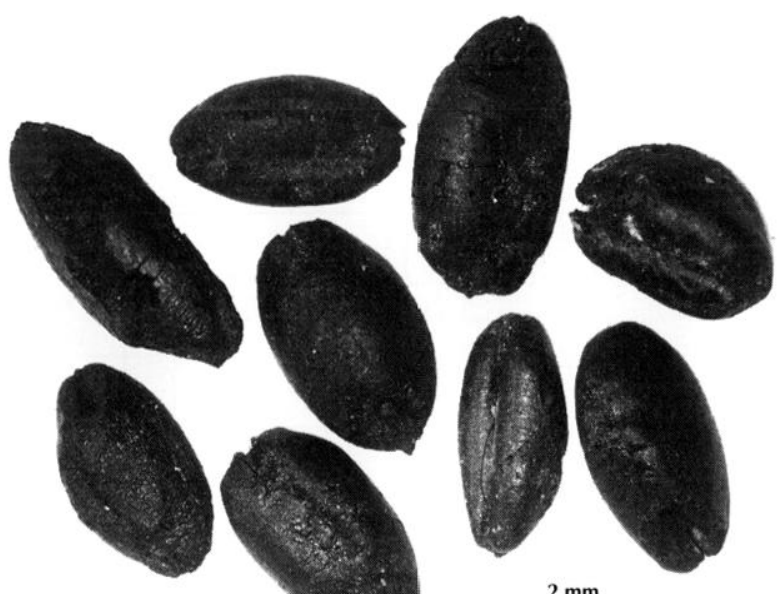

$2 \mathrm{~mm}$

1
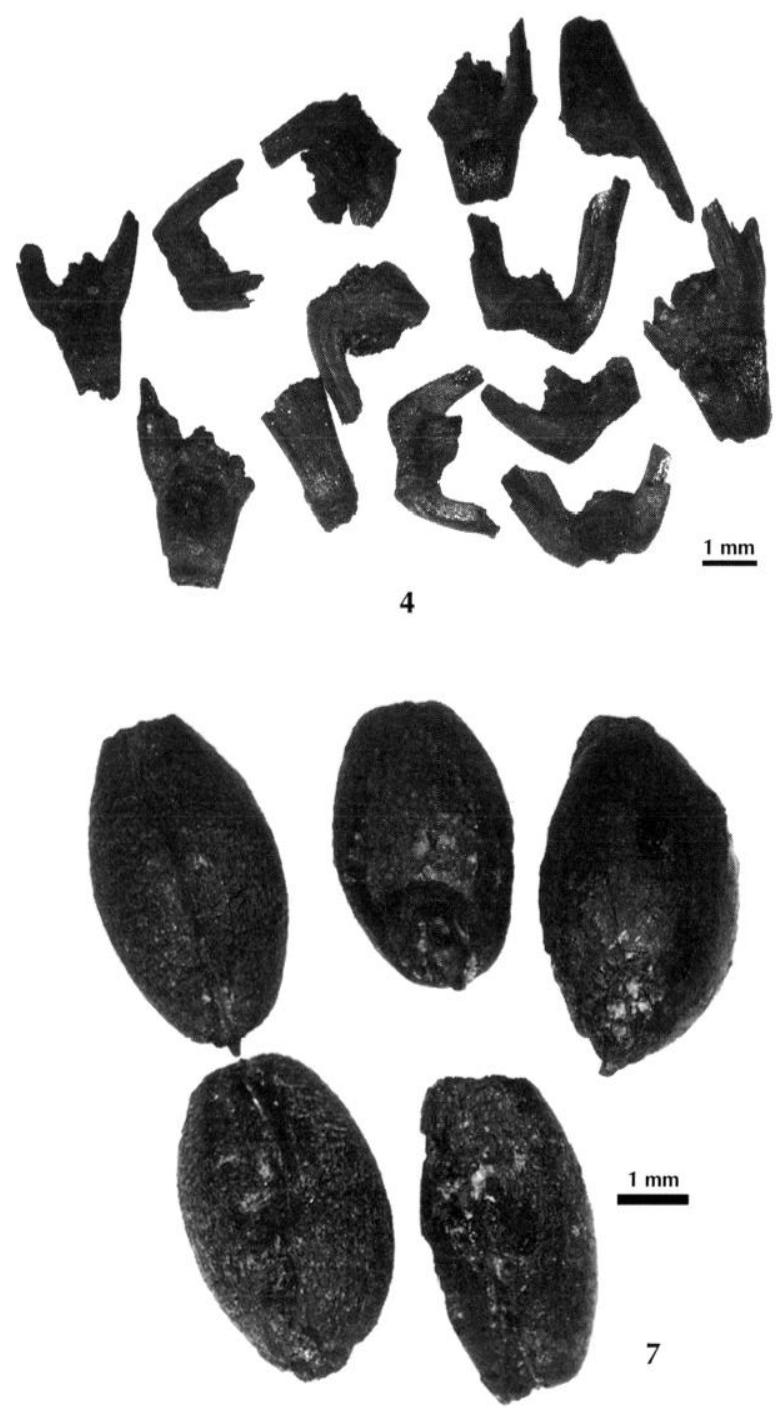

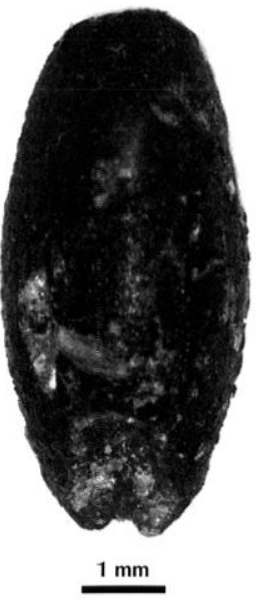

2
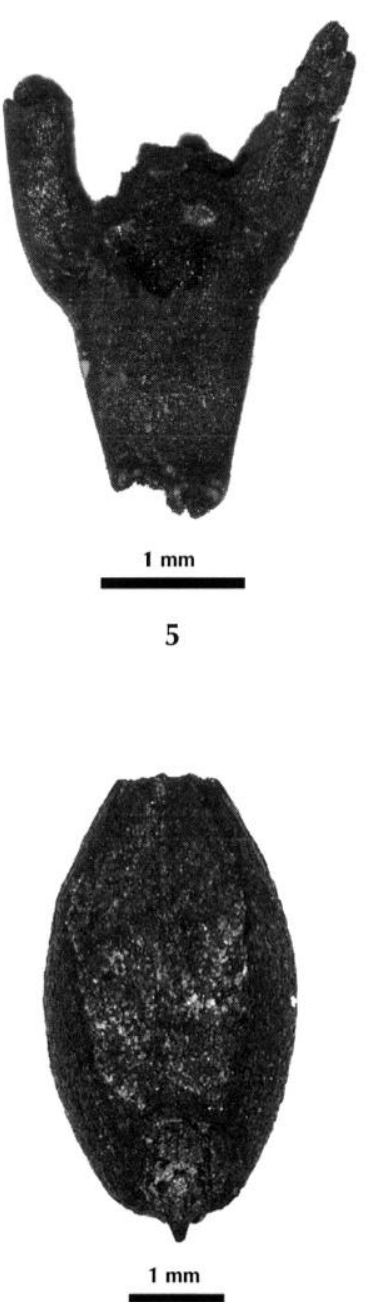

8

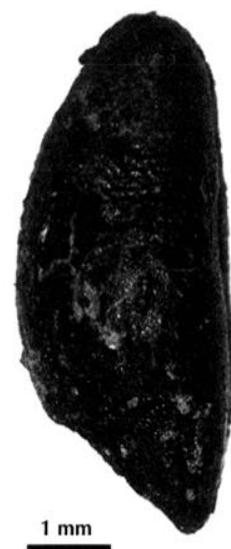

3

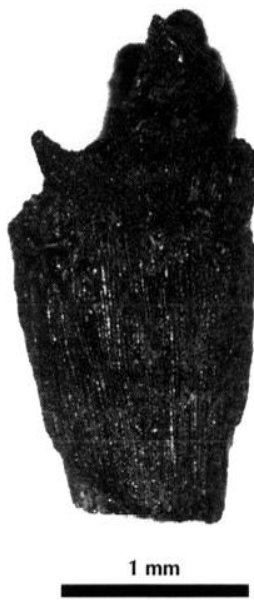

6

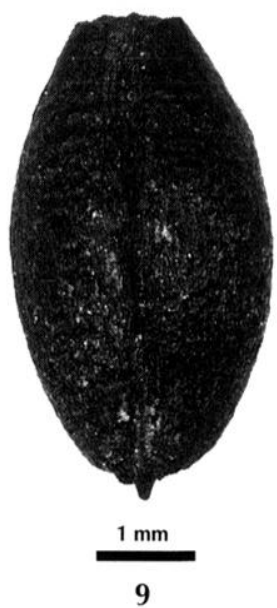

Fig. 3 - Restes de céréales carbonisés de la grotte de Foissac : 1, 2, 3, caryopses d'amidonnier (Triticum dicoccum) 4, 5, furca d'amidonnier; 6, entrencud de blé nu (Triticum aestivum s.l.) ; 7, 8, 9, caryopses d'orge polystique nue (Hordeum vulgare var. nudum). 
Tabl. II - Résultats carpologiques du secteur du Chaos de la grotte de Foissac, empreintes dans l'argile cuite ( $F$ de $T$. : front de taille).

\begin{tabular}{|l|l|c|c|c|c|c|r|}
\hline Carré & & F. de T. & J31A & M19 & N19 & N80 & Total \\
\hline Taxons attestés & & & & & & & \\
\hline Hordeum vulgare var. nudum & sem. & - & - & - & 10 & - & $\mathbf{1 0}$ \\
\hline Triticum dicoccum & sem. & 1 & - & 4 & - & - & 5 \\
\hline & épillet & 2 & - & - & - & 1 & 3 \\
\hline & fg. épi & - & 1 & - & - & - & 1 \\
\hline Total & & 3 & $\mathbf{1}$ & $\mathbf{4}$ & $\mathbf{1 0}$ & $\mathbf{1}$ & $\mathbf{1 9}$ \\
\hline
\end{tabular}

où elle dépasse généralement les $50 \%$, taux atteint exceptionnellement dans les autres carrés. Malgré une certaine variabilité à l'intérieur des échantillons, la conservation est de façon générale assez mauvaise. Les grains sont souvent gonflés ou alvéolés, stigmates laissés par une carbonisation à haute température et/ou avec un apport d'air important (Boardman, Jones, 1990).

Les taxons les plus fréquents et les mieux représentés en nombre de restes sont les céréales, très nettement dominées par le blé amidonnier (Triticum dicoccum). Celui-ci est accompagné de l'orge polystique nue (Hordeum vulgare var. nudum), du blé nu (Triticum aestivum/turgidum) et du blé engrain (Triticum monococcum). Chaque espèce est à la fois représentée par des grains et par des éléments de l'épi (vannes) : entrenœuds, furca et bases de glumes (fig. 3). Notons que les caractères morphologiques des trois entrenouuds de blé nu retrouvés dans les carrés J 21 et K 20 plaident pour une attribution à une forme hexaploïde, de type froment (Triticum aestivum s.l.) (fig. $3, \mathrm{n}^{\circ}$ 6). Parmi les fragments de grains de céréales non identifiés (Cerealia), certains éléments présentent des cassures émoussées qui montrent que la fragmentation a eu lieu avant la carbonisation (fig. $4, \mathrm{n}^{\circ} 1$ ) ; elle ne résulte donc pas de processus taphonomiques. L'orge nue et l'amidonnier sont également identifiés par les empreintes imprimées dans l'argile cuite. La prcmic̀rc cspèce est uniquement représentée par des caryopses isolés alors que la seconde est attestée par des grains, des épillets et un fragment d'épi.

Deux espèces s'ajoutent aux céréales pour compléter le corpus des plantes domestiques : le lin usuel (Linum usitatissimum) (fig. $4, \mathrm{n}^{\text {os }} 3$ et 4 ) et le pois (Pisum sativum), représenté par une seule graine. Le lin est attesté par un nombre relativement élevé de semences, ainsi que par un fragment de capsule. La caméline (Camelina sativa) est considérée comme une plante sauvage, pour des raisons qui seront discutées plus loin (fig. $4, \mathrm{n}^{\mathrm{os}} 6$ et 7 ).
Les plantes sauvages sont principalement représentées par des espèces herbacées (15 taxons au minimum). Le noisetier (Corylus avellana) constitue la seule essence ligneuse. Dans la végétation actuelle, les herbacées identifiées se rencontrent dans différentes unités écologiques. L'ensemble le mieux représenté est celui des pelouses et prairies qui rassemble l'aigremoine eupatoire (Agrimonia eupatoria), le bugle (Ajuga sp.), l'avoine élevée (Arrhenatherum elatius ssp. bulbosus), le gaillet cf. caille-lait blanc (Galium cf. mollugo), la fléole des prés (Phleum pratense) et éventuellement le brome mou (Bromus hordeaceus) (fig. 4, $\mathrm{n}^{\circ} 5$ ). Les messicoles se limitent au gaillet gratteron/à trois pointes (Galium aparine/tricorne), peut-être au brome fauxseigle (Bromus secalinus), et à la caméline (Camelina sativa s.l.), qui affectionne aussi particulièrement les cultures de lin. La renouée des oiseaux (Polygonum aviculare), le sureau yèble (Sambucus ebulus) et le chénopode blanc (Chenopodium album) témoignent de milieux rudéralisés, la dernière espèce étant surtout fréquente dans les cultures sarclées. L'oseille cf. sanguine (Rumex cf. sanguineus) (fig. $4, \mathrm{n}^{\circ} 8$ ) et la laîche (Carex sp.) peuvent être considérés comme des indicateurs de sols humides.

Les échantillons du Chaos ont en outre livré quelques fragments d'une matière organique carbonisée de nature non identifiée. Un élément contenait notamment des fragments de grains de blé pris dans la matrice amorphe (fig. $4, \mathrm{n}^{\circ} 2$ ). La présence de ces fragments pourrait indiquer qu'une partie de cette matière organique provient d'une préparation alimentaire. Un autre exemplaire recelait des éléments de tiges de plantes herbacées dont le caractère cylindrique, creux, relativement robuste et portant de fines nervures parallèles plaide pour une attribution aux graminées.

Plusieurs échantillons contenaient un nombre important de coprolithes carbonisés. Ces derniers possèdent une forme cylindrique, une longueur de $3,79 \mathrm{~mm}$ en moyenne pour un diamètre moyen de $1,44 \mathrm{~mm}$ (tabl. III),

Tabl. III - Données métriques sur les coprolithes de rongeurs carbonisés du secteur du Chaos de la grotte de Foissac (mesures effectuées sur 50 individus).

\begin{tabular}{|l|c|c|}
\hline & Longueur & Diamètre \\
\hline Moyenne & 3,79 & 1,44 \\
\hline Écart-type & 0,72 & 0,31 \\
\hline Minimum & 2,61 & 0,84 \\
\hline Maximum & 5,41 & 2,30 \\
\hline
\end{tabular}



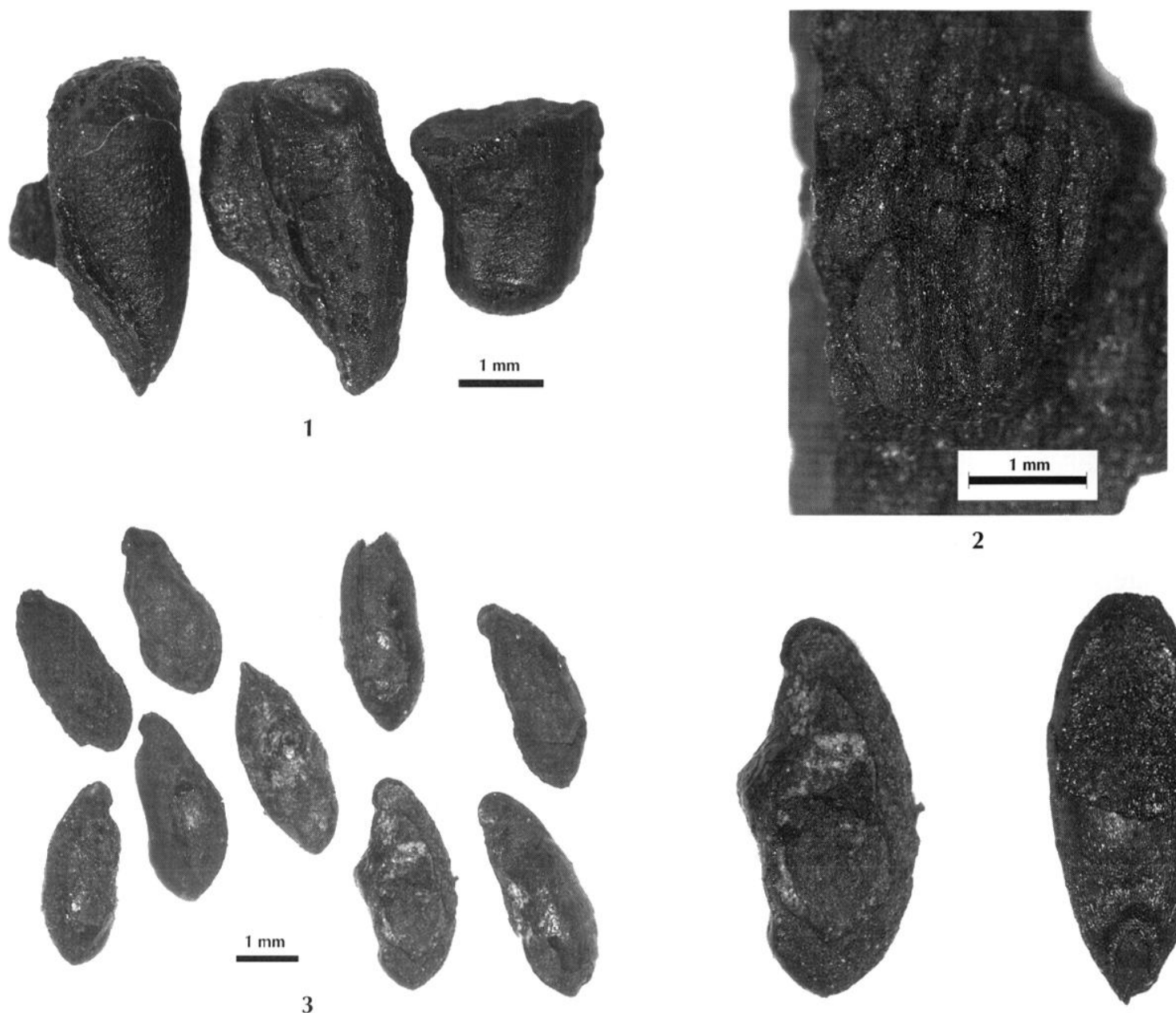

2

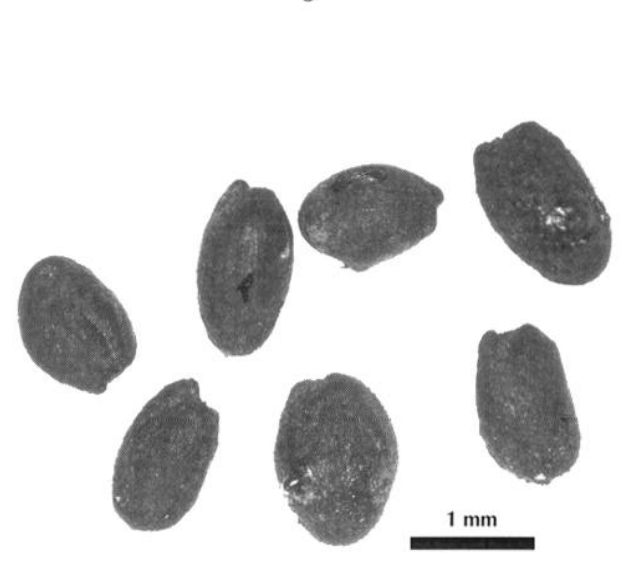

6
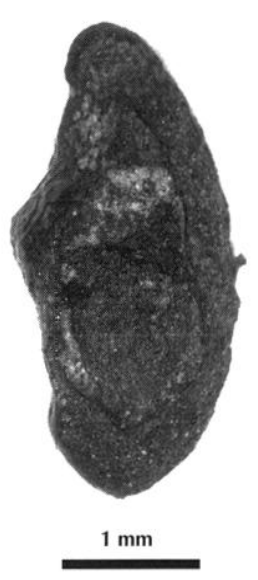

4

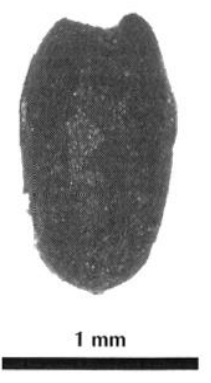

7
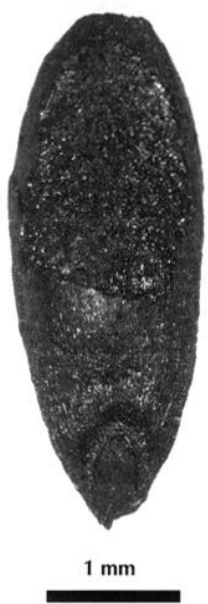

5

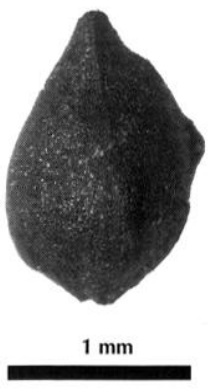

8

Fig. 4 - Restes carpologiques carbonisés provenant du secteur du Chaos de la grotte de Foissac: 1, fragments de caryopses de céréales brisés avant carbonisation ; 2, matière organique carbonisée (príparation alimentaire?) contenant des fragments de grains de blé (Triticum sp.) ; 3, 4, graines de lin cultivé (L inum usitatissimum) ; 5, brome mou/faux-seigle (Bromus hordeaceus/secalinus) ; 6, 7, caméline (Camelina sativa) ; 8, patience of. sang-de-dragon (Rumex of. sanguineus). 


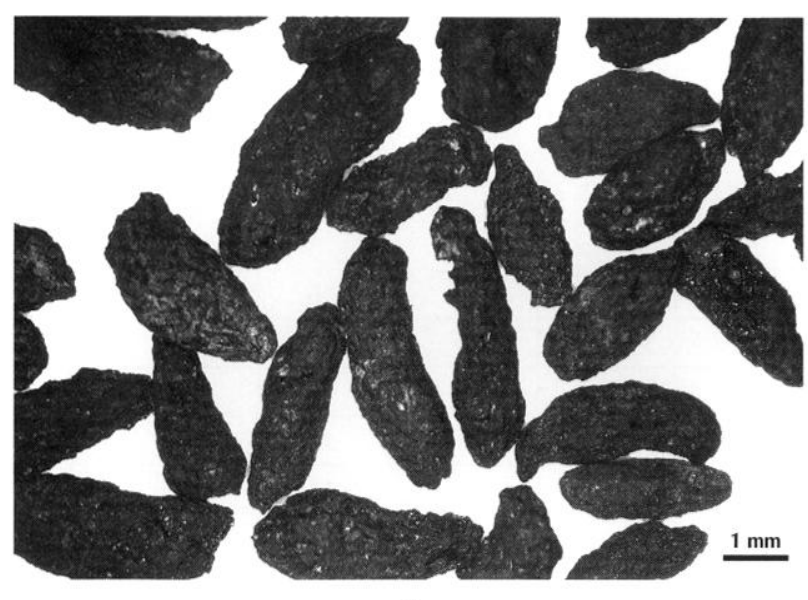

1
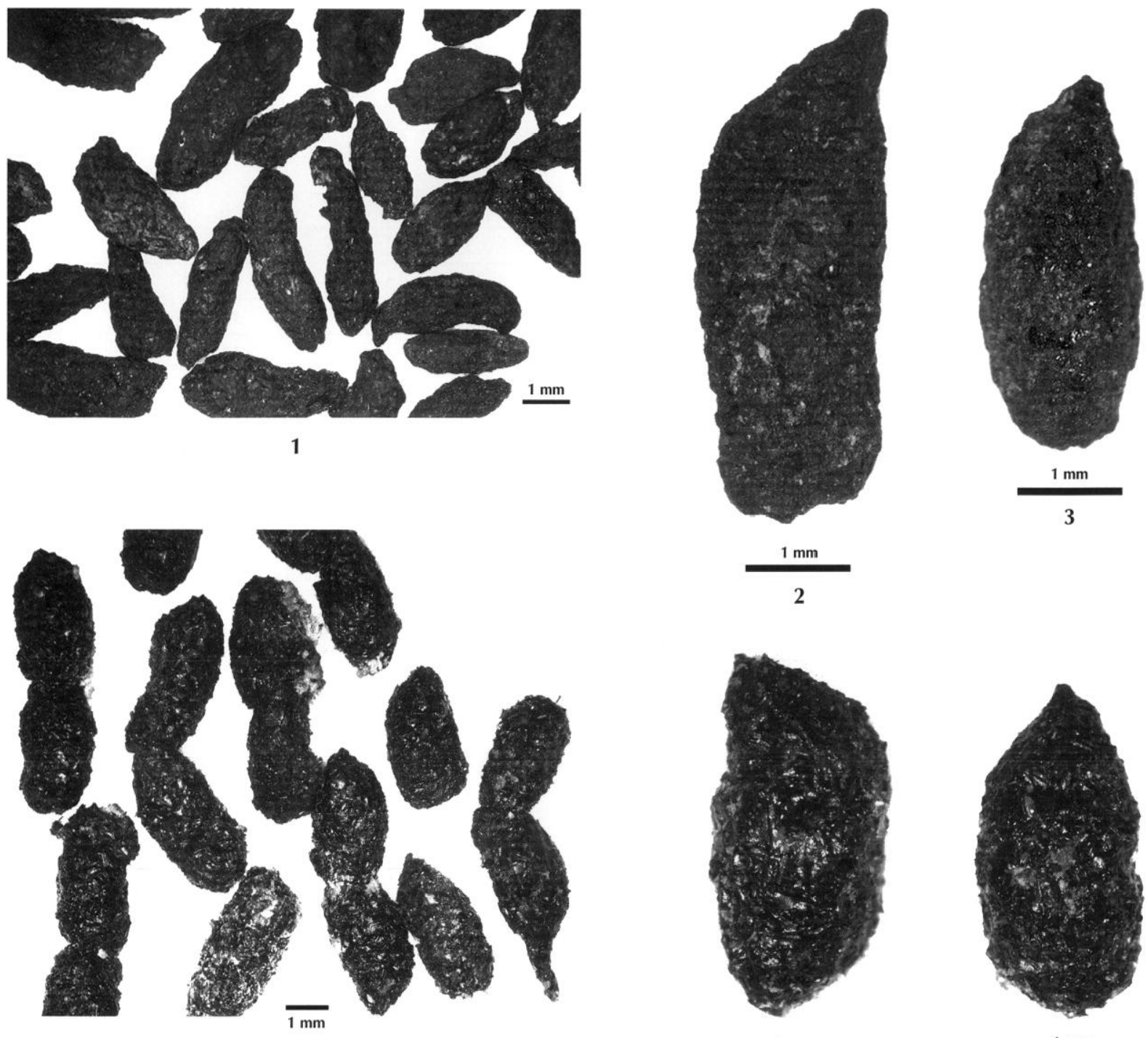

4
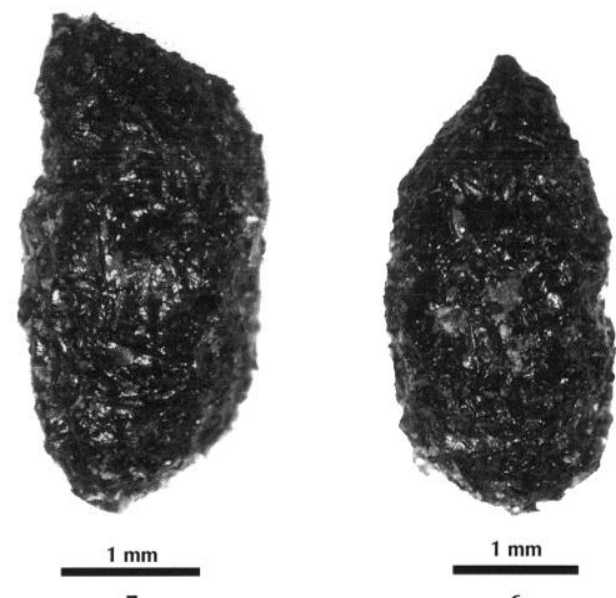

5

6

Fig. 5 - Excréments de rongeurs : 1, 2, 3, coprolithes carbonisés provenant du secteur du Chaos de la grotte de Foissac; 4, 5, 6, déjections de souris (Mus musculus) prélevées à l'intérieur d'une réserve actuelle de grains de céréales.

une ou les deux extrémités pouvant s'achever par un léger ombilic en pointe (fig. 5). Ces caractéristiques conduisent à attribuer ces coprolithes à un rongeur. L'état carbonisé des crottes et leur présence à l'intérieur d'une couche d'incendie, donc liée à un événement ponctuel dans le temps, dominée par les grains de céréales, conduit à penser qu'il pouvait exister une relation de cause à effet entre la présence des céréales et celle des coprolithes. S'il s'avère que les céréales du secteur du Chaos constituent les restes de réserves incendiées, la présence de coprolithes de rongeurs en leur sein constituerait un très intéressant et précoce témoignage d'infestation des stocks de céréales par les rongeurs. Il est de ce fait indispensable de prendre en considération les coprolithes dans l'étude de la répartition et de l'organisation des produits végétaux dans le secteur du Chaos.

\section{LE SECTEUR DE LA RIVIÈRE}

Dans le secteur de la Rivière, le nombre de restes recueillis et de taxons identifiés est nettement moindre (tabl. IV). Grâce aux conditions anaérobies propres au lit de la rivière, des semences non carbonisées (imbibées) sont ici observées au côté des restes pyrolysés. 
Tabl. IV - Résultats carpologiques du secteur de la Rivière de la grotte de Foissac.

\begin{tabular}{|c|c|c|c|c|c|c|c|c|}
\hline Carré & & X96 & $\mathrm{X} 98 / \mathrm{C} 4$ & Y99/C2 & Y99/C4 & Y99/C5 & Total & Ubiquité (Nb) \\
\hline Taxons attestés & type reste & & & & & & & \\
\hline \multicolumn{9}{|l|}{ Restes carbonisés } \\
\hline Cerealia & fg. & - & 1 & - & 2 & - & 3 & 2 \\
\hline \multirow[t]{2}{*}{ Hordeum vulgare var. nudum } & sem. & - & - & 1 & 5 & - & 6 & 2 \\
\hline & fg. & - & - & 1 & 1 & - & 2 & 2 \\
\hline Triticum aestivum/turgidum & sem. & - & 1 & - & 9 & - & 10 & 2 \\
\hline Triticum dicoccum & sem. & - & 2 & 1 & 14 & - & 17 & 3 \\
\hline Triticum sp. & fg. & - & - & - & 12 & - & 12 & 1 \\
\hline cf. Lathyrus cicera/sativus & fg. & - & - & - & 1 & - & 1 & 1 \\
\hline Galium cf. aparine & sem. & - & - & - & 4 & - & 4 & 1 \\
\hline \multirow[t]{2}{*}{ Prunus spinosa } & sem. & - & - & - & 1 & - & 1 & 1 \\
\hline & fg. & - & - & - & 1 & - & 1 & 1 \\
\hline \multicolumn{9}{|l|}{ Restes non carbonisés } \\
\hline Corylus avellana & fg. & 1 & - & - & - & 1 & 2 & 2 \\
\hline Crataegus sp. & sem. & - & - & - & 1 & - & 1 & 1 \\
\hline \multirow[t]{2}{*}{ Prunus spinosa } & sem. & - & - & - & 1 & - & 1 & 1 \\
\hline & fg. & - & - & - & 1 & - & 1 & 1 \\
\hline \multirow[t]{2}{*}{ Rubus fruticosus } & sem. & - & - & - & 11 & - & 11 & 1 \\
\hline & fg. & - & - & - & 4 & - & 4 & 1 \\
\hline Sambucus ebulus & sem. & - & - & - & 11 & - & 11 & 1 \\
\hline Sambucus nigra & sem. & - & - & - & 3 & - & 3 & 1 \\
\hline \multirow[t]{2}{*}{ Vitis vinifera ssp. sylvestris } & sem. & - & 3 & - & 31 & - & 34 & 2 \\
\hline & fg. & - & - & - & 2 & - & 2 & 1 \\
\hline Total & & 1 & 7 & 3 & 115 & 1 & 127 & \\
\hline
\end{tabular}

Les exemplaires carbonisés font largement écho aux déterminations effectuées dans le secteur du Chaos. On retrouve les mêmes céréales, à l'exception de l'engrain qui n'est pas mentionné ici. L'amidonnier occupe toujours la position dominante. Une seconde légumineuse potentiellement cultivée, la gesse (cf. Lathyrus cicera/satizus), semble présente dans la Rivière sous la forme d'un unique fragment de graine.

Les restes de prunelles (Prunus spinosa) carbonisés renvoient au matériel imbibé, intégralement composé de fruits d'arbustes et de buissons (fig. 6) : noisetier (Corylus avellana), aubépine (Crataegus sp.), prunellier, ronce (Rubus fruticosus), sureaux (Sambrucus ebulus, S. nigra) et vigne sauvage (Vitis vinifera ssp. sylvestris). Quelques akènes carbonisés de gaillet grateron (Galium cf. aparine) constituent les seules traces de plantes herbacées sauvages.

Il faut considérer que la faiblesse du nombre de restes récoltés et du nombre de taxons identifiés n'est pas repré- sentative de l'assemblage archéologique. L'enregistrement quasi exclusif de taxons producteurs de grosses semences confirme que le tri des tamis réalisé directement sur le chantier a conduit à tronquer le corpus taxinomique.

\section{RÉPARTITION DES PRODUITS VÉGÉTALX ET DES COPROLITHES DE RONGEURS DANS LE SECTEUR DU CHAOS}

Une étude de la répartition des carporestes doit permettre de vérifier s'il existe ou non des zones de concentration de certains produits végétaux et, ainsi, de confirmer ou d'infirmer l'hypothèse de l'existence de stockages dans le secteur du Chaos. La prise en considération des coprolithes de rongeurs dans cette analyse devrait conduire à vérifier si ces derniers sont bien liés à des stocks céréaliers. 

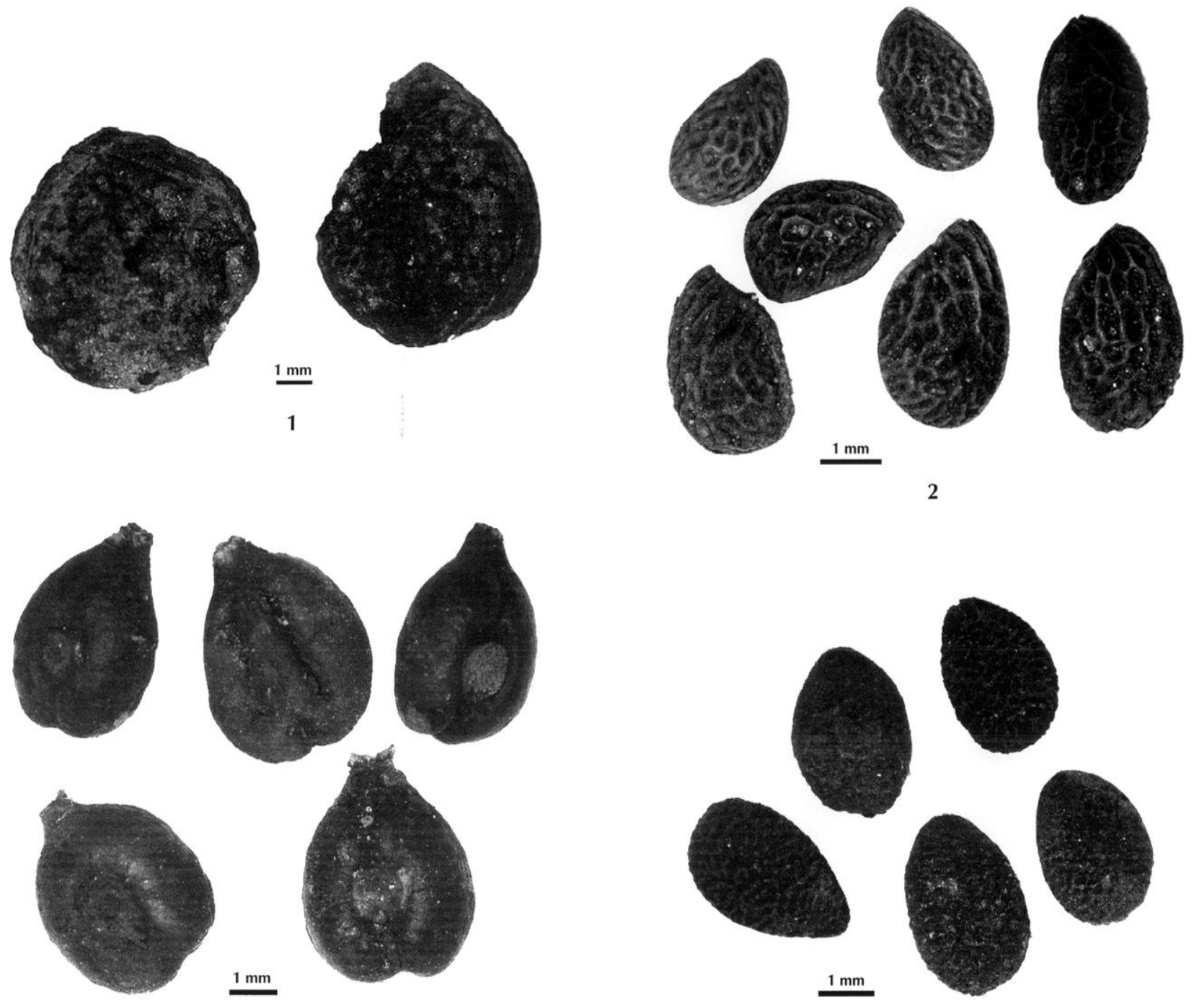

3

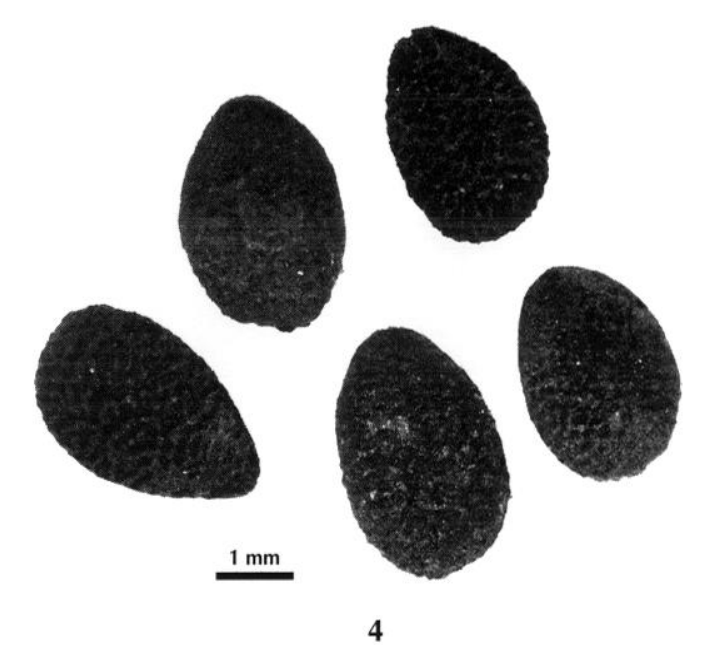

Fig. 6 - Restes carpologiques non carbonisés provenant du secteur de la Rivière dans la grotte de Foissac : 1, prunellier (Prunus spinosa) ;

2, roncier mûrier (Rubus fruticosus) ; 3, vigne sauvage (Vitis vinifera ssp. sylvestris) ; 4, sureau yèble (Sambucus ebulus).

Le volume de sédiment tamisé n’étant pas systématiquement connu, la répartition des carporestes ne peut pas être évaluée selon leur densité dans le sédiment ; seul le nombre de restes absolu est exploitable. Afin d'atténuer quelque peu les effets de la fragmentation, le nombre de restes utilisé correspond au nombre de restes entiers additionné de la moitié du nombre de fragments (fig. 7).

Deux zones de concentration apparaissent clairement : d'une part les carrés M 19, M 20 et, dans une moindre mesure, L 19, L 20 ; d'autre part les carrés K 20 et surtout $\mathrm{K} 21$, J 20 et J 21. Une évaluation de la densité carpologique est disponible pour un échantillon de la première zone, confirmant l'existence de la concentration (2 236 restes/l en $M$ 19). En revanche, le chiffre obtenu en J 20 (12 restes/l) ne semble pas confirmer l'existence de la seconde concentration. Toutefois cet échantillon fut prćlcvé essentiellement dans le niveau d'argile sous-jacent; il ne permet donc pas une évaluation correcte. Les deux zones de concentration correspondent bien aux endroits où deux grands vases de stockage écrasés en place ont été observés par les fouilleurs et déjà mis en relation avec une abondance particulière de semences carbonisées (J-K 20-21 et L-M 18-19).

La confirmation de l'existence de ces deux concentrations renforce l'hypothèse de la conservation de produits végétaux à l'intérieur des vases. Il faut toutefois considérer 
Fig. 7 - Répartition du matériel carpologique et des coprolithes dans le secteur du Chaos de la grotle de Foissac. Diagrammes établis d'après le nombre de restes (NR). Pour les semences, $N R=$ nombre d'individus $+1 / 2$ nombre de fragments. Pour les vannes, $N R=$ nombre équivalent de semences (ex. 1 furca $d e T$. dicoccum $=2$ semences).

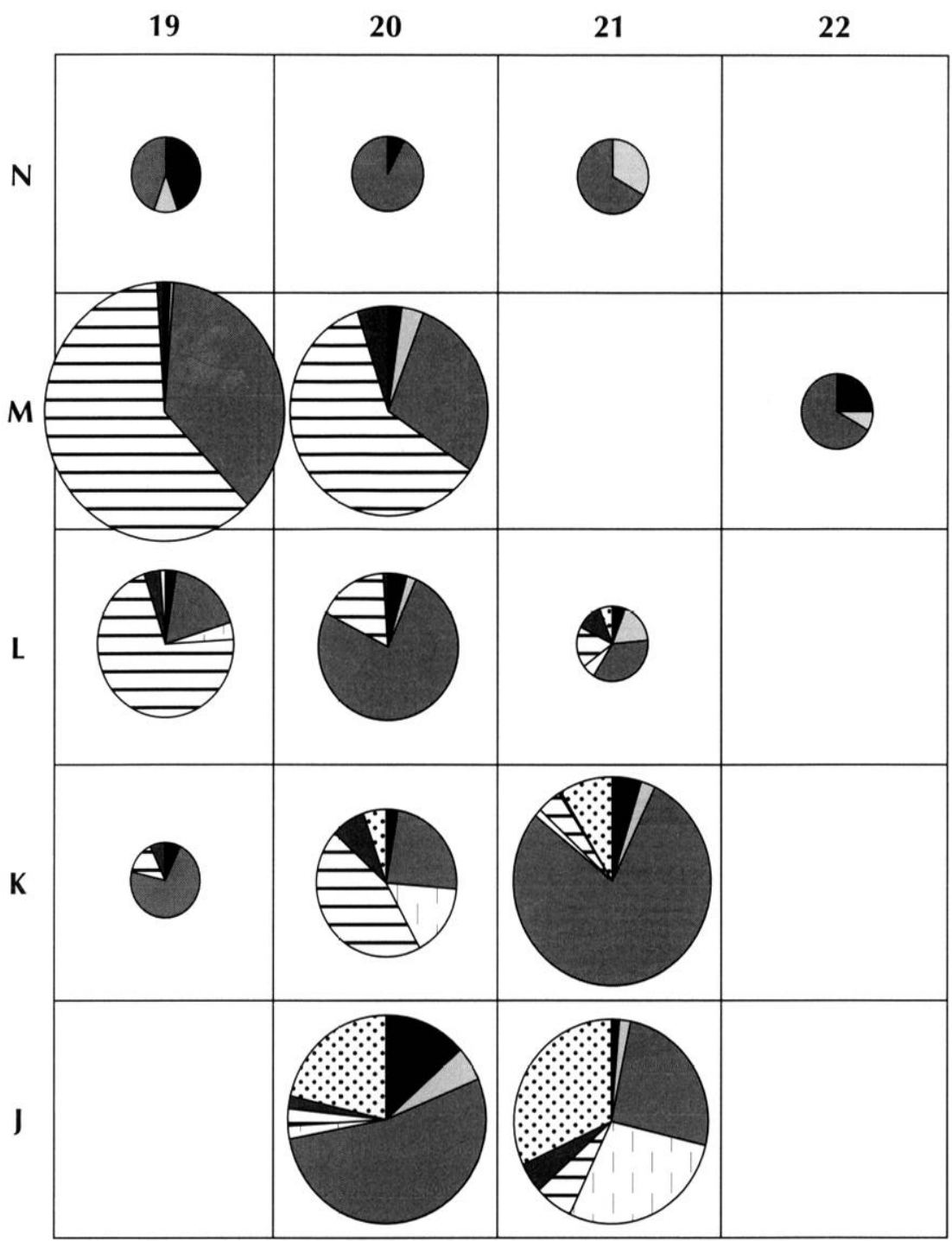

Hordeum vulg. var. nudum

Triticum dicoccum

Triticum aestivum/turgidum

Linum usitatissimum

vannes

sauvages

coprolithes

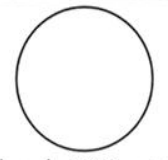

plus de 500 restes

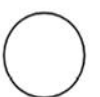

de 25 à 100 restes

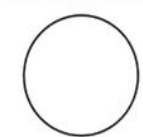

de 100 à 500 restes

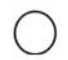

moins de 25 restes que le soutirage au travers des blocs a pu entraîner la disparition d'une partie des restes végétaux en certains endroits, voire accentuer leur concentration ailleurs. Toutefois l'hypothèse de lots indépendants de produits végétaux est nettement confirmée par une certaine structuration dans la distribution des restes carpologiques.
La concentration des carrés L-M 19-20 est très largement dominée par les restes d'amidonnier, grains et plus particulièrement vannes (furca et bases de glumes). L'engrain semble associé à l'amidonnier en proportion très minoritaire. Le total des vannes constitue $16,3 \%$ de l'assemblage carpologique de $\mathrm{L} 20$ et dépasse toujours $60 \%$ dans les trois 


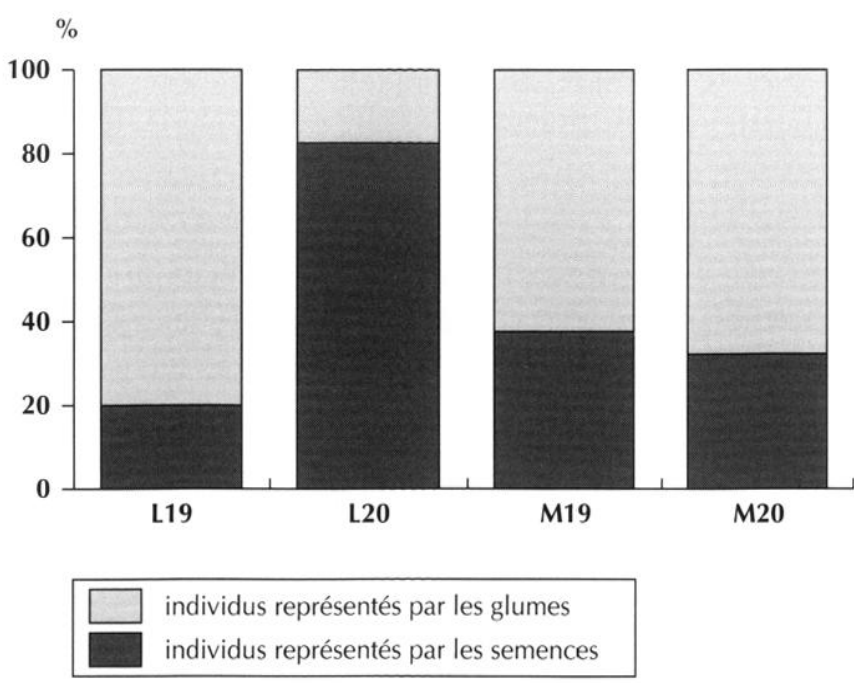

Fig. 8 - Rapport entre le nombre d'individus d'amidonnier (Triticum dicoccum) et d'engrain (T. monococcum) représentés par les glumes et par les semences dans la concentration carpologique des carrés L-M 19-20 du secteur du Chaos de la grotte de Foissac.

autres carrés. En ce qui concerne spécifiquement l'amidonnier et l'engrain, il existe une nette surreprésentation des bases de glumes par rapport au nombre de grains (fig. 8), excepté dans le carré L 20 qui constitue probablement une zone périphérique où l'épandage se mélange à d'autres ensembles. En revanche, la domination des vannes s'étend dans le carré voisin $\mathrm{K} 20$. Cette surreprésentation des vannes est d'autant plus remarquable que l'on sait que ces éléments résistent moins bien que les grains à la carbonisation (Boardman, Jones, 1990). Les brisures de céréales dont la fragmentation est antérieure à la carbonisation se concentrent également dans les carrés L-M 19.

Dans l'amas de J-K 20-21, si l'on excepte l'importance des vannes en K 20 , vraisemblablement liée à l'épandage de l'ensemble précédemment évoqué, trois traits marquants se dégagent. On remarque tout d'abord le rôle dominant que jouent toujours les grains d'amidonnier, en particulier dans les carrés J 20 et $\mathrm{K} 21$. En revanche, les vannes sont ici beaucoup plus discrètes; elles représentent au plus entre $5 \%$ et $20 \%$ des grains identifiés. On constate ensuite que les graines de lin se concentrent dans cette même zone, notamment en K 20 et J 21. L'intégralité des graines de caméline fut également recueillie dans ces deux carrés. Il est donc permis de supposer qu'il existe une relation entre les deux plantes.

Le dernier caractère marquant de la concentration J-K 20-21 est constitué par la localisation préférentielle en son sein des coprolithes de rongeurs, représentant plus de $20 \%$ des restes en J 20 et J 21.

\section{DISCUSSION}

\section{LES PRODUITS VÉGÉTAUX ENTREPOSÉS DANS LA GROTTE}

Malgré l'incendie et les perturbations taphonomiques, en particulier les soutirages liés à l'activité de la Jonquière, deux aires de concentrations de carporestes carbonisés sont toujours observables dans le secteur du Chaos ; elles représentent au moins l'épandage de deux lots distincts de produits végétaux. La composition des assemblages peut fournir de précieuses indications sur leur nature.

Le premier ensemble (L-M 19-20) apparaît peu perturbé (fig. 7) ; il est largement dominé par l'amidonnier, l'engrain constituant de toute évidence une simple « contamination " de ce dernier. Il s'agit vraisemblablement d'une adventice tolérée. L'élément marquant est la forte proportion occupée par les vannes qui, en dépit d'une probable distorsion taphonomique défavorable, témoignent d'un nombre de grains supérieur à celui réellement attesté par les semences. Les vannes constituent des déchets éliminés lors du traitement des céréales; leur importance indique que le matériel végétal constituant le premier assemblage correspond à un sous-produit du traitement de l'amidonnier plutôt qu'au produit primaire. Dans les chaînes traditionnelles du traitement des blés vêtus, les furca et bases de glumes sont essentiellement éliminées lors des opérations de nettoyage (vannage et surtout tamisage fin) succédant au décorticage (Hillman, 1981 ; Hillman, 1984a). La présence de brisures de grains fragmentés avant carbonisation doit également être interprétée comme résultant du décorticage. De tels fragments pourraient constituer les produits d'une préparation alimentaire de type gruau ou boulgour (Valamoti, 2002). Toutefois, la pratique expérimentale du décorticage a montré que cette opération entraînait involontairement la fragmentation d'une proportion variable de grain, que les outils utilisés soient la meule et la mollette ou le mortier et le pilon (Meurers-Balke, Lüning, 1992). Compte tenu de la faible proportion de grains cassés et de leur présence dans un assemblage carpologique dominé par des vannes constituant des déchets de décorticage, cette dernière hypothèse apparait bien comme la plus probable. La production de grains brisés au cours du décorticage des blés vêtus a d'ailleurs déjà été attestée dans d'autres sites 
archéologiques du midi de la France, en particulier dans la grotte du Bronze final II de Baume Layrou, Gard (Bouby, 2003 ; Bouby et al., 2005 ; Bouby, Fages, sous presse).

Un sous-produit du traitement de l'amidonnier était donc conservé dans un vase de la grotte de Foissac. Ces produits secondaires possèdent de multiples utilisations dans les sociétés agricoles non mécanisées, actuelles ou passées : fourrage, combustible, matériau artisanal (pour la fabrication de torchis par exemple), nourriture humaine de seconde qualité ou de famine (Hillman, 1981, 1984a, 1984b ; Peña-Chocarro, 1999). Le sous-produit de la grotte de Foissac contient une proportion importante de grain (nettoyage imparfait ou partiel ?), ce qui augmente d'autant sa qualité pour servir d'aliment, humain ou animal. Mais, au-delà de cette observation, il cst impossible de préciser pour quel(s) usage(s) les hommes du Chalcolithique ont souhaité le conserver. Il a pu être produit à l'extérieur, puis apporté dans la grotte pour une mise en réserve ou une utilisation locale. Toutefois, le décorticage de l'amidonnier a également pu avoir lieu sur place. La cavité a livré un nombre important de meules (Cours et al., 1984) qui, potentiellement, peuvent être utilisées à cet effet, bien que l'usage du mortier-pilon semble plus efficace (Hillman, 1984b). Le décorticage à la meule possède en particulier l'inconvénient d'entraîner la fragmentation d'une proportion dc grain plus élevée (Meurers-Balke, Lüning, 1992) : la présence de cassures de grains à Foissac pourrait-elle alors être considérée comme un indice confirmant l'utilisation des meules pour le décorticage?

La composition de l'autre assemblage (carrés J-K 20-21) est moins homogène (fig. 7) ; il représente au moins le mélange de deux produits différents. La domination générale de l'amidonnier montre qu'un lot de cette céréale était également conservé dans ce secteur, sans doute à l'intérieur du grand vase de stockage écrasé sur place. Les graines dc lin, fortement concentrées dans les carrés K 20 et J 21, ne sont probablement pas associées au blé vêtu et constituent plutôt un lot distinct mélangé au premier après l'incendie. Les fouilleurs ont observé dans cette zone l'existence d'autres vases, de contenance plus petite (inférieure à un litre), brisés in situ (Cours et al., inédit). Le lin était sans doute contenu dans l'un ou plusieurs d'entre eux.

L'ensemble d'amidonnier est de nature différente du précédent. Dans le cas présent, les grains dominent largement aux dépens des vannes, de telle sorte que l'amidonnier contenu dans le vase semble décortiqué et nettoyé.
Il peut dès lors être tentant de considérer cet ensemble comme le produit complémentaire du sous-produit conservé dans l'autre vase de stockage. On a toutefois signalé, d'une part quc lcs cffcts de la carbonisation étaient particulièrement sensibles dans les bandes I-J-K, d'autre part que l'action du feu peut introduire un biais taphonomique défavorable aux vannes (Boardman, Jones, 1990). Le décorticage du second lot d'amidonnier doit donc demeurer à l'état d'hypothèse.

\section{LES DÉGÂTS CAUSÉS PAR LES RONGEURS}

Les coprolithes associés à cette réserve de grains d'amidonnier représentent très certainement les déjections de rongeurs ayant parasité et souillé le stockage. Aujourd'hui, les principaux rongeurs ravageurs des greniers sont la souris (Mus musculus) et les rats, rat noir (Rattus rattus) et rat gris ou surmulot (Rattus norvegicus) (Cruz et al., 1988). Parmi ces espèces, la petite taille des coprolithes de Foissac (tabl. III) conduit à rejeter l'hypothèse des rats. La longueur moyenne des coprolithes archéologiques est de $3,79 \mathrm{~mm}$, alors que la longueur des excréments de rats serait au minimum de $9 \mathrm{~mm}$ pour le rat noir et $12 \mathrm{~mm}$ pour le rat gris (Preben, Dahlström, 1985 ; Schilling et al., 1986). En outre, l'établissement des rats en Europe occidentale ne semble pas confirmé avant le deuxième âge du Fer, voire l'Antiquité, pour le rat noir, et pas avant l'époque moderne en ce qui concerne le surmulot (Armitage, 1994; Audoin-Rouzeau, Vigne, 1994).

En revanche, le calibre et la forme des coprolithes archéologiques peuvent correspondre à ceux de la souris (fig. 5). Cette dernière produit des crottes de $6 \mathrm{~mm}$ de long environ, sur $2 \mathrm{~mm}$ à $2,5 \mathrm{~mm}$ de diamètre (Preben, Dahlström, 1985 ; Schilling et al., 1986). Si l'on tient compte de la rétraction vraisemblablement causée par l'action du feu, ces dimensions pourraient s'accorder au calibre moyen des coprolithes archéologiques (3,79 $\mathrm{mm} \times 1,44 \mathrm{~mm}$; tabl. III). Cependant, l'hypothèse attribuant ces déjections à la souris est en contradiction avec les données archéozoologiques actuelles sur la migration de l'animal vers l'ouest du Bassin méditerranéen et de l'Europe. Effectivement, même si quelques attestations anciennes ont parfois été proposées, comme dans les niveaux néolithiques de Font Juvénal, Aude (Marquet, 1987), même si un déficit méthodologique dans la collecte et l'identification des ossements entraîne une mauvaise connaissance des phénomènes de diffusion de l'espèce (Cucchi et al., 2002, 2005), une 
révision critique de la documentation existante montre que la présence dans le sud-ouest de l'Europe de la souris domestique, commensale de l'homme, n'est pour l'heure pas attestée, et semble peu probable, avant l'âge du Fer (Cucchi et al., 2005).

S'il ne faut peut-être pas exclure totalement la présence de la souris domestique dans le sud-ouest de la France au Chalcolithique, il faut considérer que d'autres espèces de rongeurs, aujourd'hui plus rares ou absentes dans les greniers, pouvaient occuper cette niche écologique avant l'arrivée de la souris et des rats. Il convient de citer en particulier le mulot sylvestre (Apodemus sylvaticus) et le mulot à collier (A. flavicollis) (Auffray et al., 1990 ; Vigne, 1997; Cucchi et al., 2005). Le mulot sylvestre est omnivore et consomme notamment des semences de graminées. Il possède de fortes capacités d'acclimatation quant à son habitat; on le rencontre généralement dans les bois, les haies, les champs, les jardins et à l'occasion en situation commensale, pénétrant dans les bâtiments occupés par les hommes, particulièrement en hiver, surtout si la souris est absente du biotope (Burton, 1976; Mourier, Winding, 1979 ; Schilling et al., 1986; Mistrot, 2000). Ses crottes semblent légèrement plus courtes et de forme relativement plus massive que celle de la souris (Preben, Dahlström, 1985); elles pourraient donc également constituer une attribution plausible pour les coprolithes archéologiques. L'hypothèse d'une occupation de la niche écologique commensale de l'homme par le mulot avant l'arrivée de la souris en Europe a déjà été formulée par les archéozoologues et la présence d'Apodemus flavicollis dans les maisons $\mathrm{du}$ Néolithique final des villages lacustres de Clairvaux-MM et de Chalain 3 (Jura) semble bien attestée (Vigne, 1997). L'occupation première de la niche écologique commensale par le mulot aurait même constitué un des principaux obstacles à la migration de la souris vers l'ouest du Bassin méditerranéen, jusqu'à l'établissement des premières grandes agglomérations au premier millénaire av. J.-C., donnant un avantage compétitif à cette dernière (Cucchi et al., 2005).

Qu'elles proviennent de la souris domestique ou d un mulot, les nombreuses déjections retrouvées à l'intérieur d'un stock céréalier de la grotte de Foissac constituent une preuve directe - et à notre connaissance unique - de l'occupation la niche écologique commensale par les rongeurs dès le Chalcolithique et des dégâts que pouvaient causer ces derniers dans les stockages alimentaires. Il n'est pas possible de préciser si la contamination excrémentielle s'est produite dans la grotte même, ce qui pourrait signifier qu'elle constituait un lieu de stockage habituel, ou à l'extérieur, lors d'une éventuelle étape de stockage préalable. Quoi qu'il en soit, l'ampleur des dégâts apparaît très importante: le rapport entre coprolithes et grains de céréales oscille entre $7 \%$ et $52 \%$ dans les carrés J 20, J 21 et K 21 . Si l'amidonnier contenu dans le vase représente bien le produit primaire, une telle pollution indiquerait qu'il s'agit sans doute des restes d'un stock ancien, vraisemblablement pas de la dernière récolte mais plutôt de celle d'un été antérieur. Dans les greniers à riz de Taiwan, l'âge du stock est considéré comme un paramètre déterminant pour la taille des populations de souris (Mus musculus castaneus) qui les colonisent (Chou et al., 1998). Une autre hypothèse doit cependant être envisagée : le vase pouvait receler un sousproduit du nettoyage de l'amidonnier, résultat d'un tri visant précisément à l'élimination des excréments. Mais c'est alors la part du grain éliminé qui paraîtrait exagérément élevée, rendant sans doute cette conjecture peu plausible.

L'abondance des coprolithes conduit à s'interroger sur le caractère consommable ou non du produit pour les humains. Il faut bien sûr considérer que les standards de l'hygiène chalcolithique étaient vraisemblablement éloignés des pratiques actuelles. D'ailleurs, pour la période romaine, de multiples mentions archéologiques de stocks de céréales endommagés par l'humidité ou souillés par les insectes parasites existent en Europe ; la consommation de grain en partie gâté ne devait pas être rare (Matterne et al., 1998). Sans doute en allait-il de même aux époques antérieures. Les excréments de rongeurs ne semblent pas toxiques en eux-mêmes mais ces animaux sont vecteurs de diverses maladies (peste, rage, leptospirose, hantavirus, salmonellose, campylobactériose, cryptosporidiose, toxoplasmose, listériose). En outre, leur activité a dû causer d'importantes pertes quantitatives dans le stock d'amidonnier. À titre d'exemple, une souris domestique adulte mange environ $3 \mathrm{~g}$ de nourriture solide par jour, soit 70 à 100 grains de blé, et en endommage une quantité encore supérieure car elle ingère rarement en totalité les grains rongés (Mourier, Winding, 1979). Or, la présence de grains cassés, dont l'endosperme peut directement être attaqué, favorise le développement de moisissures (Proctor dir., 1994). Par ailleurs, si leur activité s'est déroulée dans la grotte, les rongeurs ont pu s'attaquer aux produits carnés, à la structure en bois du Chaos, ainsi qu'aux conteneurs en vannerie vraisemblablement présents dans ce secteur (Cours et al., inédit). 


\section{PLANTES COMPAGNES, ADVENTICES ET PRATIQUES AGRICOLES}

À l'inverse de l'amidonnier et du lin, aucune concentration particulière de blé nu et d'orge nue n'est observable. Ces taxons sont répartis sur toute la surface étudiée du Chaos. Il ne peut être exclu que de petites concentrations aient existé dans certains des carrés non étudiés ou que celles-ci soient trop perturbées par l'incendie et les soutirages pour être encore aujourd'hui perceptibles. On peut cependant supposer que, comme l'engrain, l'orge et le blé nu étaient associés à l'amidonnier, représentant des contaminations (sans doute) tolérées dans les champs, possibles rémanences de cultures antérieures. Par ailleurs, on a pu constater l'association étroite entre lin et caméline, qui ne constitue pas plus de $10 \%$ de l'ensemble. Cette dernière représente donc une mauvaise herbe du lin plutôt qu'une plante cultivée indépendante ou volontairement mélangée à celui-ci. Aucun indice solide de mise en culture de la caméline n'existe d'ailleurs en Europe avant l'âge du Bronze. La mention de Foissac constitue, associée à celle du Néolithique moyen du Vieil-Auzay (Vendée), les plus anciennes traces en Europe occidentale de cette plante oléagineuse dont la diffusion dans les campagnes européennes s'est probablement réalisée comme mauvaise herbe avant sa domestication (Bouby, 1998).

Bien que les plantes sauvages des pelouses et prairies représentent l'ensemble écologique le plus diversifié, elles ne forment pas de concentration de semences, susceptible d'indiquer la présence d'une réserve ou d'un épandage de fourrage. À l'exception de l'avoine élevée (Arrhenatherum elatius ssp. bulbosus) tous les taxons se rattachent aux carrés M 19-20. Ils sont toujours attestés par un nombre très faible de semences. Comme les autres herbacées sauvages, les plantes de pelouses et prairies constituaient très probablement des mauvaises herbes des espaces cultivés. Elles furent apportées dans la grotte avec leurs hôtes, en l'occurrence l'amidonnier puisqu'elles se trouvent à l'intérieur des déchets de décorticage. Les espèces de pelouses et prairies présentes sont essentiellement des hémicryptophytes. Un travail du sol léger, comme celui pratiqué à l'araire, favorise le mélange de telles plantes bisannuelles ou pérennes avec les espèces annuelles classiques des communautés actuelles de mauvaises herbes (Hillman, 1991).

Dans les chaines traditionnelles de traitement des céréales vêtues, une proportion très importante des mauvaises herbes est éliminée lors des opérations de vannage et de tamisage qui succèdent au décorticage (Hillman, 1981, 1984a). Or, il faut souligner le nombre très limité de semences d'adventices recueillies à l'intérieur des déchets de décorticage des carrés L-M 19-20. Une aussi faible contamination malherbologique semble impliquer, soit un désherbage exceptionnellement soigneux des parcelles, soit la sélection d'une partie seulement des mauvaises herbes par coupe haute de la récolte, soit la séparation des épis de la paille avant le battage. À l'exception du bugle (Ajuga sp.), dont la croissance ne dépasse pas $40 \mathrm{~cm}$, toutes les plantes sauvages associées aux déchets de décorticage peuvent atteindre des hauteurs de $80 \mathrm{~cm}$ et plus. Cet élément conduit à privilégier l'hypothèse d'une récolte haute, sans exclure la séparation des seuls épis dans un second temps après moisson par coupe basse. Notons qu'un magnifique exemplaire de "couteau de moissonneur " a été trouvé dans la grotte, à proximité de la sépulture 1, et un second hors stratigraphie (Cours et al., 1984 ; Garcia et al., 1987). L'usage d'un tel instrument pour la récolte des céréales s'accorde parfaitement avec l'hypothèse d'une coupe haute (Alonso i Martínez, 1999).

\section{LES PLANTES VIVRIÈRES DU CAUSSE AU CHALCOLITHIQUE}

Les carporestes du secteur du Chaos représentent un ensemble de produits présents au moment de l'incendie qui a figé leur enregistrement pour des millénaires. Il n'est toutefois pas possible du seul fait de leur conservation de savoir s'ils étaient d'usage courant ou anecdotique, à la différence d'assemblages détritiques, comme celui de la Rivière, qui ont toutes les chances d'être constitués de l'accumulation de déchets multiples, et donc de mieux refléter la succession des pratiques quotidiennes les plus communes.

On peut supposer que l'amidonnier était une céréale d'importance pour les paysans chalcolithiques du causse de Limogne, d'autant que celui-ci semble dominant dans le secteur de la Rivière comme dans celui du Chaos. Il est en outre vraisemblable que les céréales sporadiquement mêlées à l'amidonnier dans les produits entreposés (orge nue, blé nu et engrain) étaient par ailleurs cultivées. Effectivement, ces 4 espèces constituent les céréales les plus courantes au Néolithique final et au Chalcolithique dans le sud de la France (Marinval, 1988). Dans la région quercynoise, orge nue et blé nu figurent, aux côtés de l'orge vêtue (Hordeum vulgare), dans un ensemble dominé par les vannes d'amidonnier au Néolithique final sur le site de Fontanes à 
Gaches, Lot (Bouby, 2002). Amidonnier, blé nu et, semblet-il, orge nue sont déjà présents au Néolithique moyen dans le Lot, à la Perte du Cros, Saillac (Hopf, 1967). Il est également envisageable que les deux légumineuses attestées à Foissac par des graines isolées, le pois et la gesse (cf. Lathyrus cicera/sativus), aient fait l'objet d'une exploitation agricole. Malheureusement, la région du causse de Limogne souffre d'une totale déficience d'information sur la culture des légumineuses à la fin du Néolithique.

La présence d'un petit assemblage de graines de lin constitue un apport important. Jusqu'à présent, malgré la présence sporadique de semences dans quelques sites, il n'avait pas été possible de démontrer la culture de cette plante oléagineuse et textile au Néolithique dans le sud et l'ouest de la France (Ruas, Marinval, 1991), alors que celleci était clairement établie dans les sites lacustres de la zone alpine au Néolithique final (Lundström-Baudais, 1986). Cette lacune était sans doute en grande partie due au déficit de tamisages fins dans les anciennes analyses carpologiques, mais également au fait que le lin est plus fréquemment enregistré dans les assemblages humides, sous la forme de graines non carbonisées. Or, ce type de milieu de conservation fait largement défaut pour le Néolithique européen en dehors de la zone alpine.

Une grande variété de fruits sauvages est attestée à Foissac, principalement dans le secteur de la Rivière: aubépine (Crataegus sp.), noisetier (Corylus avellana), prunellier (Prunus spinosa), roncier mûrier (Rubus fruticosus), sureaux (Sambucus ebulus, S. nigra) et vigne sauvage (Vitis vinifera ssp. sylvestris). Cet enregistrement carpologique, exceptionnellement permis par les conditions anaérobies de la Rivière, laisse entrevoir le rôle que pouvait jouer la cueillette dans l'alimentation humaine.

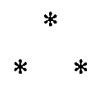

En dépit de l'incendie, des perturbations liées à l'activité de la rivière et d'un échantillonnage relativement limité, il a été possible de restituer l'existence de, au minimum, 3 types de produits végétaux mis en réserve dans le secteur du Chaos : un lot constitué de produits secondaires du décorticage de l'amidonnier, composé majoritairement de vannes, un stock ancien, pour le moins âgé de plus d'un an, d'amidonnier également, fortement contaminé par des coprolithes de rongeurs commensaux, et enfin une petite réserve de lin. Il est avéré que les deux premiers ensembles étaient conservés dans des vases de stockage, il est probable que les graines de lin se trouvaient à l'intérieur d'un ou de plusieurs petits vases, à proximité des grains d'amidonnier. Les vases étaient vraisemblablement rangés dans une structure en bois aménagée sur le Chaos rocheux, voisinant semble-t-il avec des provisions carnées (Lignereux et al., 1994). Parmi d'autres hypothèses, les archéologues ont supposé que la grotte a pu constituer un lieu de refuge ou de cachette en période de troubles (Cours et al., inédit), à l'image du schéma proposé pour la grotte des Planches-près-Arbois (Jura) à l'âge du Bronze final (Pétrequin et al., 1985). Dans cette conjecture et selon les informations dont nous disposons, les réfugiés se seraient trouvés munis de bien maigres provisions au moment de l'incendie et de l'abandon de la grotte. Entre autres hypothèses, la cavité a également pu servir de lieu de conservation en relation avec un habitat extérieur.

Le secteur de la Rivière a livré des informations complémentaires sur les fruits sauvages cueillis, qui venaient diversifier une alimentation fondée sur les plantes cultivées. La présence de ces éléments non carbonisés laisse également imaginer l'ampleur des informations que devaient contenir les niveaux de la rivière, assemblage anaérobie exceptionnel pour le sud de la France, et non perçues faute d'un protocole parfaitement adapté de collecte des restes végétaux.

\section{Remerciements}

Notre reconnaissance va tout d'abord au regretté François Rouzaud (Service régional de l'archéologie de Midi-Pyrénées) ; sans sa disponibilité et son enthousiasme intact pour les recherches menées à Foissac, cette étude n'aurait certainement pas été possible. Nous voudrions modestement dédier cet article à sa mémoire. Nous remercions la mairie de Foissac, pour son soutien financier, les membres de la SORES, en particulier Alain du Fayet de la Tour, et du Parc préhistorique de Foissac pour leur concours, ainsi que Thomas Cucchi (Muséum national d'histoire naturelle, UMR 5197 du CNRS), Michel Girard (Centre d'études Préhistoire, Antiquité, Moyen Âge, UMR $6130 \mathrm{du}$ CNRS) et Jean Vaquer (Centre d'anthropologie, UMR $8555 \mathrm{du}$ CNRS) pour leur aide documentaire. Merci aux deux relecteurs anonymes du manuscrit pour leurs remarques constructives qui nous ont permis d'améliorer cet article. 


\section{BIBLIOGRAPHIE}

\section{Alonso I MARTínez N.}

1999 : De la llavor a la farina. Els processos agricoles protohistơrics a la Catalunya occidental, Lattes, UMR 154 du CNRS (Monographies d'archéologie méditerranéenne, 4), $328 \mathrm{p}$.

\section{ARmitage P. L.}

1994 : "Unwelcome Companions : Ancient Rats Reviewed ", Antiquity, 68, 259, p. 231-240.

\section{AUdoin-Rouzeau F., Vigne J.-D.}

1994 : « La colonisation de l'Europe par le rat noir (Rattus rattus) ", Rerne de paléobiologie, 13, p. 125-145.

\section{AUfrRay J.-C., VANLerberghe F., Britton-} Davidian J.

1990 : "The house mouse progression in Eurasia: a palaeontological and archaeozoological approach ", Biological Journal of the Linnean Society, 41, p. 13-25.

\section{AufrRay J.-C., Cassaing J., Legendre $S$.}

1991 : "Anthropisation et biosphère : impact de l'homme sur la guilde des souris et les communautés de mammifères en Europe au Pléistocène et Holocène ", Bulletin de la Société préhistorique franşuise, 88, 4, p. 122-128.

\section{Boardman S., Jones G.}

1990 : "Experiments on the Effects of Charring on Cereal Plant Components", Journal of Archaeological Science, 17, p. 1-11.

\section{BOUBY L.}

1998 : " Two early finds of gold-of-pleasure (Camelina sp.) in Middle Neolithic and Chalcolithic sites in western France ", Antiquity, 72, 276, p. 391-398.

2002 : "Traitement des blés vêtus et structures de combustion: données carpologiques pour le Néolithique final et le Chalcolithique dans la région des Causses ", in Pirineus $i$ veins al $3^{\prime \prime}$ millenni AC. De la fi del neolitic a l'edat del bronze entre l'Ebre i la Garona. XII Col.loqui Internacional d'Arqueologia de Puigcerdà, 10-12 de novembre del 2000, Puigcerdà, Institut d'Estudis Ceretans, p. 357-366.

2003 : « De la récolte au stockage. Éclairages carpologiques sur les opérations de traitement des céréales à l'âge du Bronze dans le sud de la France ", in ANiderson P. C., Cummings L. S., Schippers T. K.,
SIMONel B. (DIR.), Le Traitement des récoltes : un regard sur la diversité, du Néolithique au présent, Actes des XXIII rencontres internationales d'archéologie et d'histoire d'Antibes, 17-19 oct., Antibes, éd. APDCA, p. $21-46$.

Bouby L., Fages G.

Sous presse: "Étude carpologique des réserves végétales de la grotte de Baume Layrou (Trèves, Gard). Aspects de la production agraire et fonction du site au Bronze final ", Documents d'archéologie méridionale.

\section{Bouby L., Fages G., Treffort J.-M.}

2005 : « Food storage in two Late Bronze Age caves of Southern France: palaeoethnobotanical and social implications", Vegetation History and Archaeobotany, (DOI : 10.1007/s00334-005-0079-6).

\section{BURTON M.}

1976 : Tous les mammiferes d'Europe en couleur, Paris, Elsevier-Séquoia, $256 \mathrm{p}$.

Chou C.-W., Lee P. F., Lu K.-H., YU H.-T.

1998 : " A Population Study of House Mice (Mus musculus castaneus) Inhabiting Rice Granaries in Taiwan ", Zoological Studies, 37,3 , p. 201-212.

Cours S., Duday H., Fayet de la Tour A. du, Garcia M.-A., Rouzaud F

1984 : " La grotte de Foissac ", Archeologia, 192-193, p. 92-108.

Inédit: Comptes rendus des recherches effectuées dans la grotte de Foissac (Aveyron) de 1978 à 1988, 11 rapports dactylographiés.

Cruz J.-F., Troude F., Griffon D., Hebert J.-P. 1988 : Conservation des grains en régions chaudes, Paris, ministère de la Coopération et du Développement, $545 \mathrm{p}$.

Cucchi T., Vigne J.-D., Auffray J.-C., Croft P., Peltenburg E.

2002 : «Introduction involontaire de la souris domestique (Mus musculus domesticus) à Chypre dès le Néolithique précéramique ancien (fin IX" et VIII ${ }^{*}$ millénaires av. J.-C.) ", Comptes rendus Palevol, 1, 4, p. 235-241.

Cucchi T., Vigne J.-D., Aufrray J.-C.

2005 : "First occurrence of the house mouse (Mus musculus domesticus Schwarz et
Schwarz, 1943) in the Western Mediterranean: a zooarchaeological revision of subfossil occurrences ", Biological Journal of the Linnean Society, 84, p. $429-445$

Garcia M.-A., Cours S., Duday H., Fayet de la TOUR A. DU, Rouzaud F.

1987 : "Les Chalcolithiques de la grotte de Foissac en Aveyron ", Objets et Mondes, 25, 1-2, p. 3-12.

\section{Garcia M.-A., Rouzaud F.}

1994 : "Les exploitations chalcolithiques d'argile dans la grotte de Foissac (Aveyron) ", in Binider D., Courtin J. (DIR.), Terre cuite et société: La céramique, document technique, économique, culturel, Actes des XIV rencontres internationales d'archéologie et d'histoire d'Antibes, 21-23 oct. 1992, Juan-les-Pins, éd. APDCA, p. 13-18.

Hiliman G. C.

1981 : " Reconstructing Crop Husbandry Practices from Charred Remains of Crops ", in Mercer R. (DIR.), Farming Practice in British Prehistory, Edinburgh, Edinburgh University Press, p. 123-162.

1984a : "Interpretation of archaeological plant remains: The application of ethnographic models from Turkey ", in VAN Zeist W., Casparit: W. A. (DIR.), Planis and Ancient Man. Studies in Palaeoethnobotany, Rotterdam, Balkema, p. 1-41.

1984b : « Traditional husbandry and processing of archaic cereals in recent times: the operations, products and equipment which might feature in Sumerian texts. Part I : the glume wheats ", Bulletin on Sumerian Agriculture, 1, p. 114-152.

1991 : "Phytosociology and Ancient Weed Floras: Taking Account of Taphonomy and Changes in Cultivation Methods ", in Harris D. R., Thomas K. D. (DIR.), Modelling Erological Change, London, Institute of Archaeology, University College, p. 27-40.

\section{Hopf M.}

1967 : « La station néolithique de la Perte du Cros à Saillac (Lot). III, Analyse de céréales du niveau III (Chasséen) ", Gallia Préhistoire, X, 1, p. 70-73.

JAUBERT J.

1996 : " Datations numériques de gisements de l'Aveyron ", Cahiers d'archéologie avegronnaise, 10, p. 93-97. 
Lignereux Y., Peters J., Cours S., Duday H., Garcia M., Fayet de la Tour A. du, RouzAud F.

1994 : "L'occupation chalcolithique de la grotte de Foissac (Aveyron) : les témoins archéozoologiques ", Revue médicale vétérinaire, 145,5 , p. $355-386$.

\section{LUNDSTRÖM-BAUDAIS $\mathbf{K}$.}

1986 : "Étude paléoethnobotanique de la station III de Clairvaux ", in PÉTREQuin P. (DIR.), Les Sites littoraux néolithiques de Clairvaux-les-lacs (Jura) -I- Problématique générale : l'exemple de la Station III, Paris, éd. de la Maison des sciences de l'Homme, p. $311-391$.

\section{Marinval P.}

1988 : Cueillette, agriculture et alimentation végélale de l'Épipaléslithique jusqu'au 2 âge du Fer en France méridionale. Apports palethnographiques de la carpologie, Doctorat nouveau régime, Paris, EHESS, $458 \mathrm{p}$.

\section{Marquet J.-C.}

1987 : " Les modifications de l'environnement postglaciaire en France méditerranéenne d'après les rongeurs de l'Abeurador et de Font-Juvenal ", in Gullainf J., Covrtin J., Roundi J.-L., Vtrnet J.-L. (DIR.), Premieres communautés paysannes en méditerranée occidentale, Paris, éd. du CNRS, p. 155-163.

Matterne V., Yvinec J.-H., Gemehl. D., RIQUiER C.

1998 : "Stockage de plantes alimentaires et infestation par les insectes dans un grenier incendié de la fin du $\mathrm{II}^{\circ}$ sieccle après J.-C. à Amiens (Somme) ", Rerue archéologique de Picardie, 3-4, p. 93-122.

\section{Meurers-Balke J., Lüning J.}

1992 : "Some aspects and experiments concerning the processing of glume wheats ", in ANDERsoN P. (DIR.), Préhistoire de l'agriculture: nouvelles approches expérimentales et ethnographiques, Paris, éd. du CNRS (coll. Monographies du CRA, 6), p. $341-362$.

\section{Mistrot V.}

2000 : « L.es micro-mammifères, marqueurs de l'anthropisation du milieu ", Ettudes rurales, janvier-juin 2000, 153-154, p. $195-206$.

\section{Mourier H., Winding $O$.}

1979: (ruide des petits animaux sauvages de nos maisons et jardins, Neuchâtel, Delachaux et Niestlé, 224 p.

\section{Peña-Chocarro L.}

1999 : Prehistoric Agriculture in Southern Spain during the Neolithic and the Bronze Age. The application of ethnographic models, Oxford, BAR, International Series, 818, $167 \mathrm{p}$.

Pétrequin P., Chaix L., Pétrequin A.-M., Piningre J.-F.

1985 : La Crotte des Planches-près-Arbois (Jura), Paris, éd. de la Maison des sciences de l'Homme, $273 \mathrm{p}$.

\section{Preben B., Dahlström P.}

1985: : Guide des traces d'animaux, Neuchâtel, Delachaux et Niestlé, $240 \mathrm{p}$.

\section{Proctor D. L. (DIR.)}

1994 : Grain Storage Techniques: Evolution and Trends in Developing, FAO Agricultural services Bulletin, 109, Rome, FAO, 277 p.

\section{Ruas M.-P., Marinval P.}

1991 : «Alimentation végétale et agriculture d'après les semences archéologiques (de 9000 av. J.-C. au $\mathrm{XV}^{e}$ siècle) ", in GUIlaINE J. (DIR.), Pour une archéologie agraire, Paris, Armand Colin, p. 409-439.

\section{Schilling D., Singer D., Diller H.}

1986 : Guide des mammifères d'Europe, Neuchâtel, Delachaux et Niestlé, $280 \mathrm{p}$.

VALAmoti S. M.

2002 : "Food remains from Bronze Age Archondiko and Mesimeriani Toumba in northern Greece?" Vegetation History and Archaeobotany, 11, p. 17-22.

VIGNE J.-D.

1997: " Les micromammifères au Néolithique final à Clairvaux-les-Lacs et Chalain 3. Contribution à l'histoire du commensalisme ", in PÉtrequin P. (DIR.), les Sites littoraux néolithiques de Clairvaux-les-Lacs et de Chalain (Jura) -III- Chalain station 3. 3200-2900 av. J.-C., Paris, éd. de la Maison des sciences de l'Homme, vol. 2, p. $717-722$. 